hep-th/0205068

KCL-MTH-02-10

SWAT-2002/337

\title{
A class of Lorentzian Kac-Moody algebras
}

\author{
Matthias R. Gaberdiel ${ }^{\star, a}$, David I. Olive ${ }^{\dagger, b}$ and Peter C. West ${ }^{\ddagger}, a$ \\ ${ }^{a}$ Department of Mathematics, King's College London \\ Strand, London WC2R 2LS, U.K. \\ ${ }^{b}$ Department of Physics, University of Wales Swansea \\ Singleton Park, Swansea SA2 8PP, U.K.
}

\begin{abstract}
We consider a natural generalisation of the class of hyperbolic Kac-Moody algebras. We describe in detail the conditions under which these algebras are Lorentzian. We also construct their fundamental weights, and analyse whether they possess a real principal so(1,2) subalgebra. Our class of algebras include the Lorentzian Kac-Moody algebras that have recently been proposed as symmetries of M-theory and the closed bosonic string.
\end{abstract}

May 2002

\footnotetext{
* e-mail: mrg@mth.kcl.ac.uk

$\dagger$ e-mail: D.I.0live@swansea.ac.uk

$\ddagger$ e-mail: pwest@mth.kcl.ac.uk
} 


\section{Introduction}

Since the realisation in the 1930's that the nuclear forces possessed an isotopic spin symmetry, finite dimensional Lie algebras have played an increasingly crucial role in our understanding of the fundamental laws of nature. In particular, we now believe that three of the four forces of nature are determined by local gauge symmetries with finite dimensional Lie algebras. The discovery of (infinite dimensional) Kac-Moody algebras in the late 1960s considerably enlarged the class of Lie algebras beyond that previously considered, and a subset of these, affine algebras, have played an important role in string theory and conformal field theory (for a review see for example [1]). However, until recently, no significant physical role has been found for more general Kac-Moody algebras, namely those still characterised by a symmetrisable Cartan matrix of finite size.

During the last few years it has become apparent that type II superstring theory has a (non-perturbative) description in eleven dimensional space-time in terms of M-theory [2]. Very little is known about the latter, but it would seem reasonable to suppose, given previous developments, that M-theory possesses a very large symmetry algebra. More recent work has tried to identify what some of this symmetry could be, and it has been conjectured that it includes a rank eleven Lorentzian Kac-Moody symmetry denoted $e_{11}$ $[3,4]$. Indeed, substantial fragments of this symmetry have been found in all the maximal supergravity theories in ten and eleven dimensions [4,5]. The rank eleven nature of this symmetry can be seen to be a consequence of the bosonic field content of the maximal supergravity theories and is related to Nahm's theorem that supergravity theories only exist in space-times with up to eleven dimensions [6].

Simple Lie algebras of finite dimensional or affine type are well studied and fully classified, being recognisable in terms of finite, connected Dynkin diagrams (representing their Cartan matrices), said to be of finite or affine type respectively. Despite a considerable literature on the other Kac-Moody algebras [7], knowledge of their properties is much less complete. Indeed, apart from a few cases, even the multiplicities of the various root spaces are unknown. It is possible that for many purposes the class of all Kac-Moody algebras may be too large and that the study of a well-motivated subclass may be more rewarding.

One extra class of Kac-Moody algebras that has been studied in some detail are those known as 'hyperbolic'. The Dynkin diagram of a hyperbolic Kac-Moody algebra is a connected diagram such that deletion of any one node leaves a (possibly disconnected) set of connected Dynkin diagrams each of which is of finite type except for at most one of affine type. More specifically, hyperbolic Kac-Moody algebras correspond to hyperbolic diagrams which are the diagrams of this type that are not of finite or affine type. The hyperbolic

Kac-Moody algebras have been classified, possess no more than ten nodes and a Cartan 
matrix that is Lorentzian, that is, nonsingular and endowed with exactly one negative eigenvalue. Furthermore every hyperbolic Kac-Moody algebra has a real principal so(1,2) subalgebra [8]. Since the rank of the symmetry underlying M-theory appears to be eleven it cannot be described by a hyperbolic Kac-Moody algebra.

In this paper we consider a larger class that does include the aforementioned $e_{11}$, as well as the proposed symmetry for the bosonic string, $k_{27}$ [4]. The Dynkin diagrams we shall consider are connected diagrams possessing at least one node whose deletion leaves a (possibly disconnected) set of diagrams, each of which is of finite type except for at most one of affine type. As was noted by Ruuska, [9], such diagrams are automatically Lorentzian if not recognisably of finite or affine type and include the hyperbolic ones. As we shall see, the corresponding algebras may or may not possess a real principal so(1,2) subalgebra, for example, $e_{11}$ does, but $k_{27}$ does not.

In section 2 we briefly recall the relations between Dynkin diagrams, Cartan matrices and Kac-Moody algebras, and describe more precisely the class of algebras that will be considered in this paper. The advantage of this class is that it is easy to determine the simple roots in terms of those for the reduced diagram, namely the diagram remaining when the central vertex has been deleted. The same is true of the fundamental weights (and hence the Weyl vector as it is their sum) and, to a lesser extent, the determinant of the Cartan matrix. But separate treatments must be made of the two cases that the reduced diagram possesses no affine component, or just one.

Section 3 treats the case when the reduced diagram (i.e. the Dynkin diagram for which the central node has been deleted) contains only connected components of finite type. In section 4 it is shown that the overall Dynkin diagram is Lorentzian if and only if there is precisely one affine component given that the reduced diagram is a mixture of connected components of finite and affine type. Then the expression for the determinant of the Cartan matrix simplifies considerably and can be used to identify a large class of unimodular even Lorentzian Cartan matrices. We also study the conditions under which these Lorentzian algebras may possess a real principal so $(1,2)$ subalgebra.

In section 5 we describe in detail a special subclass of constructions that seems to be of particular relevance in string theory. We also discuss more specifically the algebras that are associated to even self-dual lattices, and study the question of whether the algebras possess a real principal so(1,2) subalgebra. Section 6 describes further constructions and section 7 contains some conclusions. We have added four appendices in which various more technical points that are needed for our discussion are outlined. 


\section{A special class of Kac-Moody algebras}

First let us recall the definition of a Kac-Moody algebra [7] in terms of a generalised Cartan matrix. Suppose $A_{i j}$ is a generalised Cartan matrix with $i, j=1, \ldots, r$, where $r$ is the rank of the Kac-Moody algebra. We shall only consider generalised Cartan matrices that are symmetric. They satisfy

$$
A_{i i}=2,
$$

$$
A_{i j}=A_{j i} \text { for } i \neq j \text { are negative integers or zero. }
$$

Because the off diagonal entries, (2.2) could possibly take values $-2,-3$ etc, and it is not appropriate to refer to the case in which $A$ is symmetric as being simply-laced, unless the matrix is of finite or affine type and such values are disallowed. The entries of the matrix $A_{i j}$ can be encoded in terms of an unoriented graph with $r$ nodes, whose adjacency matrix is given by $2 \delta_{i j}-A_{i j} .{ }^{\star}$ This graph is called the Dynkin diagram, and it specifies the matrix $A_{i j}$ uniquely (up to simultaneous relabelling of the rows and columns). If the diagram is disconnected, the Cartan matrix has a block diagonal form (when labelling is ordered suitably) and the algebra consists of commuting simple factors.

Given a generalised Cartan matrix, the Kac-Moody algebra can be formulated in terms of a set of Chevalley generators $H_{i}, E_{i}$ and $F_{i}$ for each $i=1, \ldots, r$. These can be identified with the generators of the Cartan subalgebra, and the generators of the positive and negative simple roots, respectively. The Chevalley generators are taken to obey the Serre relations

$$
\begin{gathered}
{\left[H_{i}, H_{j}\right]=0,} \\
{\left[H_{i}, E_{j}\right]=A_{i j} E_{j},} \\
{\left[H_{i}, F_{j}\right]=-A_{i j} F_{j},} \\
{\left[E_{i}, F_{j}\right]=\delta_{i j} H_{i},}
\end{gathered}
$$

and

$$
\left[E_{i}, \ldots\left[E_{i}, E_{j}\right] \ldots\right]=0, \quad\left[F_{i}, \ldots\left[F_{i}, F_{j}\right] \ldots\right]=0 .
$$

In equation (2.7) the number of $E_{i}$ 's in the first equation, and the number of $F_{i}$ 's in the second is $1-A_{i j}$. The remaining generators of the Kac-Moody algebra are obtained as multiple commutators of the $E_{i}$ 's and as multiple commutators of the $F_{i}$ 's, using the above Serre relations. The generalised Cartan matrix therefore determines the Kac-Moody

* The $(i, j)$ entry of the adjacency matrix of an unoriented graph describes the number of links between the nodes $i$ and $j$. Our conventions here differ slightly from those of Kac [7]. 
algebra uniquely. Although this procedure is fairly simple in principle, explicit descriptions for all generators of a Kac-Moody algebra are only available for a few, rather special, algebras.

A convenient basis for the Kac-Moody algebra consists of one for the Cartan subalgebra (which has dimension $r$ ) consisting of the Chevalley generators $H_{i}$ with $i=1, \ldots, r$, and the step operators for roots. The roots are eigenvectors of the Cartan generators (under the adjoint action), and we can therefore think of them as vectors in an $r$-dimensional vector space. There exists a scalar product $($, ) on this space such that the Cartan matrix is given in terms of the simple roots as

$$
A_{i j}=\left(\alpha_{i}, \alpha_{j}\right)
$$

This means that, if the Cartan matrix $A$ is non-singular, its signature and rank is the same as that of the scalar product. If $A$ is singular there is an analogous but more complicated statement. Thus (2.1) implies that all the simple roots have length $\sqrt{2}$ and, in particular, are space-like. Their integer span is an even lattice, known as the root lattice, denoted $\Lambda_{R}(A)$. The root lattices of inequivalent Kac-Moody algebras may or may not be equivalent; indeed, we shall encounter examples of inequivalent Kac-Moody algebras (that are described by inequivalent Dynkin diagrams) with the same root lattice.

Only if the Cartan matrix $A$ is positive definite is the associated algebra of finite dimension. Then the diagram and Cartan matrix is said to be of finite type. If $A$ is positive semi-definite it is said to be of affine type. We shall mainly be interested in those Kac-Moody algebras that are Lorentzian, namely those whose generalised Cartan matrix is non-singular and possesses precisely one negative eigenvalue.

We have already mentioned the fully classified subset known as 'hyperbolic' KacMoody algebras. In this paper we will study a larger class of Kac-Moody algebras which includes the finite, affine and hyperbolic types and is automatically Lorentzian if neither of finite nor affine type. These correspond to a Dynkin diagram possessing at least one node whose deletion yields a diagram whose connected components are of finite type except for at most one of affine type.

We shall call the overall Dynkin diagram $C$, and the selected node, whose deletion yields the reduced diagram $C_{R}$, the "central" node. For many examples the central node is not uniquely determined by the property that $C_{R}$ has only connected components of finite and affine type, and what we shall do in the following will apply to every admissible choice for the central node. Unlike the overall $C$, the reduced diagram $C_{R}$ need not be connected. If it is disconnected denote the $n$ connected components $C_{1}, C_{2} \ldots C_{n}$. The 
Cartan matrix of $C_{R}$ is obtained from that for $C$ simply by deleting the row and column corresponding to the central node. Then the overall Dynkin diagram can be re-constructed from the reduced Dynkin diagram and the central node, denoted $c$, by adding those edges linking the latter to each node of $C_{R}$. The number of links of the central node $c$ to the $i$ 'th node is

$$
\eta_{i}=-A_{c i},
$$

namely the entries in the row and column of the Cartan matrix of $C$ whose deletion was just mentioned.

Note that if the connected components of $C_{R}$ are all of finite or affine type, the offdiagonal elements in its Cartan matrix only take the values 0 or -1 (for convenience $A_{1}^{(1)}$ in standard notation is excluded). This limitation does not apply to the values of $\eta_{i}$ which could be any integer $0,1,2 \ldots$. Schematically, the Dynkin diagram $C$ has the structure:

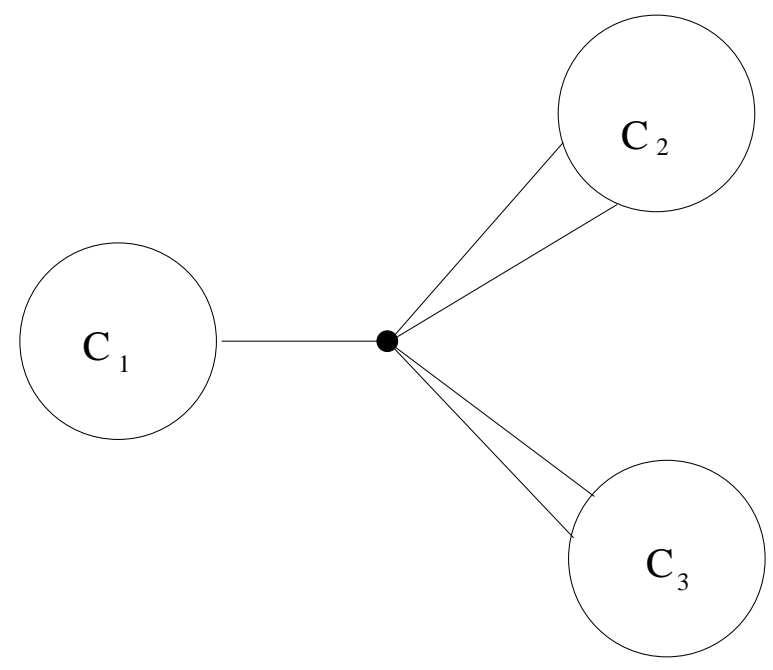

Fig. 1: The Dynkin diagram $C$ of the Kac-Moody algebra $\mathfrak{g}$ for the case $n=3$.

Notice that the diagram $C$ need not be a tree diagram and indeed may possess any number of loops.

If the Cartan matrix $A$ is non-singular it is possible to define fundamental weights $\lambda_{1}, \lambda_{2}, \ldots \lambda_{r}$ reciprocal to the simple roots

$$
\lambda_{i}=\sum_{j=1}^{r}\left(A^{-1}\right)_{i j} \alpha_{j}, \quad \lambda_{i} \cdot \alpha_{j}=\delta_{i j} .
$$

For hyperbolic algebras these all lie inside or on the same light-cone (so that the entries $\left(A^{-1}\right)_{i j}=\lambda_{i} \cdot \lambda_{j}$ are all negative or zero), but for Lorentzian algebras that are not hyperbolic some fundamental weights must be space-like. 
The integer span of the fundamental weights forms a lattice known as the weight lattice $\Lambda_{W}(A)$. It is reciprocal to the root lattice $\Lambda_{R}(A)$ yet contains it. Hence it is possible to consider the quotient which forms a finite abelian group $\mathrm{Z}(A)$ containing $|\mathrm{Z}(A)|$ elements

$$
\Lambda_{W}(A) / \Lambda_{R}(A)=\mathrm{Z}(A) .
$$

Of course this group is encoded in the Cartan matrix whose determinant, up to a sign, equals the number of elements it contains

$$
\operatorname{det} A= \pm|\mathrm{Z}(A)|
$$

If $A$ is of finite type it is associated with a finite dimensional semi-simple Lie algebra $\mathfrak{g}$ which can be exponentiated uniquely to a simply connected Lie group $G$ whose centre is the finite abelian group $\mathrm{Z}(A)$.

Given the fundamental weights, the Weyl vector $\rho$ also exists

$$
\rho=\sum_{i=1}^{r} \lambda_{i}=\sum_{i, j=1}^{r}\left(A^{-1}\right)_{i j} \alpha_{j}
$$

satisfying the fundamental property

$$
\rho . \alpha_{i}=1, \quad i=1,2 \ldots r .
$$

A critical question for the existence of a principal so(1,2) subalgebra with desirable reality properties is whether the coefficients $\sum_{i=1}^{r}\left(A^{-1}\right)_{i j}$ in (2.12a) are all of the same sign or not. More precisely, by a real principal so $(1,2)$ subalgebra is meant one that has standard hermiticity properties given the hermiticity properties of the Kac-Moody algebra. This has desirable consequences for unitarity, and more details and explanations are given in appendix A. It leads to more stringent conditions than those discussed by Hughes [10]. We shall only consider real principal so(1,2) subalgebras in this paper, and we shall therefore drop from now on the qualifier 'real'. In the finite case the coefficients $\sum_{i=1}^{r}\left(A^{-1}\right)_{i j}$ are all positive, while they are all negative in the hyperbolic case and in the Lorentzian case mixed signs are possible. We shall show that, within the class defined above, one of these signs at least is negative. 


\section{When the reduced diagram is of finite type}

In this case we shall construct linearly independent simple roots for the overall Dynkin diagram $C$ in terms of those for the reduced diagram $C_{R}$ as well as doing the same for the fundamental weights when that is possible. A formula for the determinant of the Cartan matrix of $C$ will likewise be found.

The $r-1$ simple roots for $C_{R}, \alpha_{1}, \alpha_{2}, \ldots \alpha_{r-1}$, are linearly independent and span a Euclidean space of dimension $r-1$ since the reduced diagram $C_{R}$ is of finite type. They will suffice for the corresponding nodes of the overall Dynkin diagram $C$ once they are augmented by the simple root for the central node

$$
\alpha_{c}=-\nu+x, \quad \text { where } \quad \nu=\sum_{i=1}^{r-1} \eta_{i} \lambda_{i}=-\sum_{i=1}^{r-1} A_{c i} \lambda_{i} .
$$

Here $\lambda_{i}$ are the fundamental weights (2.10) for the reduced diagram (which is assumed to have a non-singular Cartan matrix) and lie in the space spanned by the simple roots, while $x$ is a vector orthogonal to that space. Evidently this guarantees $\alpha_{i} . \alpha_{c}=A_{i c}$ leaving only the condition

$$
2=A_{c c}=\nu^{2}+x^{2}
$$

which determines the sign of $x^{2}$. If $C_{R}$ is of finite type (whether disconnected or not), its simple roots span a Euclidean space. Thus the simple roots of $C$ span a space that is Euclidean or Lorentzian according as $x^{2}$ is positive or negative. Likewise if $x^{2}$ vanishes the simple roots span a space with a positive semi-definite metric and so constitute an affine root system. Since Dynkin diagrams of finite or affine type are fully classified they are recognisable as such. Hence if $C$ is of neither of these types it must be Lorentzian, as claimed earlier. It is at this stage that the connection (2.8) between the scalar product and the Cartan matrix is exploited.

The $r$ fundamental weights for the overall diagram $C$ will be denoted $\ell_{c}, \ell_{1}, \ell_{2}, \ldots \ell_{r-1}$ in order to distinguish them from the $(r-1)$ fundamental weights $\lambda_{1}, \ldots, \lambda_{r-1}$ for the reduced diagram. They are related to each other by

$$
\ell_{i}=\lambda_{i}+\frac{\nu \cdot \lambda_{i}}{x^{2}} x, \quad \ell_{c}=\frac{1}{x^{2}} x,
$$

providing $x^{2}$ does not vanish, that is the overall diagram $C$ is not affine. This accords with the fact that the definition (2.10) fails only in this case. The overall Weyl vector is then

$$
R \equiv \sum_{j=c}^{r-1} \ell_{j}=\rho+\frac{(1+\nu \cdot \rho)}{x^{2}} x,
$$


where $\rho=\sum_{j=1}^{r-1} \lambda_{j}$ is the Weyl vector for the reduced diagram, (the same as the sum of the Weyl vectors for each connected component of $C_{R}$ if it is disconnected). Notice that

$$
R . \ell_{c}=\frac{(1+\nu \cdot \rho)}{x^{2}}
$$

and hence has the same sign as $x^{2}$ given that $\nu . \rho$ is positive when $C_{R}$ is of finite type (as all quantities $\lambda_{i} \cdot \lambda_{j}$ are). Thus at least one of the coefficients in the expansion of the Weyl vector $R$ in terms of simple roots is negative when $C$ is Lorentzian.

An instructive example is provided by choosing the linking coefficients $\eta_{i}$ to equal each other, taking the value $\bar{\eta}$ say, so that the central node is linked by precisely $\bar{\eta}$ edges to each other node. Then $\nu$ in (3.1) equals $\bar{\eta}$ times the Weyl vector $\rho$ and, by (3.2), $x^{2}=2-\bar{\eta}^{2} \rho^{2}$. Hence equations (3.3) and (3.4) lead to

$$
R . \ell_{c}=\frac{\bar{\eta} \rho^{2}+1}{x^{2}}, \quad R \cdot \ell_{i}=\frac{(2+\bar{\eta}) \rho \cdot \lambda_{i}}{x^{2}},
$$

which are all negative if $x^{2}$ is, that is if $C$ is Lorentzian. In particular, this therefore means that there are infinitely many Lorentzian algebras with a principal so(1,2) subalgebra that can be obtained by this construction since we can choose $\mathfrak{g}$ to be any finite dimensional semi-simple Lie algebra. The simplest example is obtained by considering $\bar{\eta}=1$, and taking $\mathfrak{g}=\mathrm{su}(m)$ for $m \geq 4$. The Dynkin diagram for the case $m=9$ is shown below.

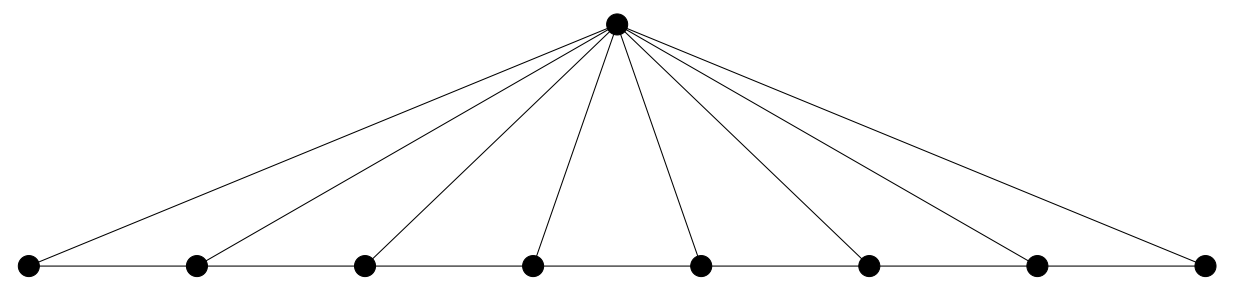

Fig. 2: The Dynkin diagram of the Kac-Moody algebra $\mathfrak{g}$ obtained by taking $\mathfrak{g}_{1}=\mathrm{su}(9)$ and $\nu=\rho$.

The determinant of the Cartan matrix $A$ for the overall diagram $C$ is related to the Cartan matrix $B$ of the reduced diagram $C_{R}$ (obtained from $A$ by deleting the row and column corresponding to the central node) by

$$
\operatorname{det} A=x^{2} \operatorname{det} B=\left(2-\nu^{2}\right) \operatorname{det} B
$$

or, using (3.1) and the fact that $\lambda_{i} \cdot \lambda_{j}=\left(B^{-1}\right)_{i j}$

$$
\operatorname{det} A=\left(2-\sum_{i, j=1}^{r-1} \eta_{i}\left(B^{-1}\right)_{i j} \eta_{j}\right) \operatorname{det} B
$$


or remembering that the adjugate of a matrix (the matrix of cofactors) equals the inverse matrix times the determinant

$$
\operatorname{det} A=2 \operatorname{det} B-\sum_{i, j=1}^{r-1} \eta_{i}(\operatorname{adj} B)_{i j} \eta_{j}
$$

This final version makes it clear that the result is indeed an integer even though the sign is unclear. Equation (3.9) also has the virtue that it makes good sense even when $B$ is singular. Indeed it simplifies considerably as the first term on the right hand side drops out. We shall return to this point in the next section.

Equation (3.7) can be proven directly by using (2.8) to factorise $\operatorname{det} A$ into products of determinants of matrices made of the components of the simple roots of $C$. The crucial point is that the simple roots for nodes of $C_{R}$ have no component in the direction of $x$ and this makes it trivial to evaluate the factored determinants. Alternatively (3.9) is just an application of Cauchy's expansion of bordered determinants [11].

Only now is account taken of the fact that the reduced diagram $C_{R}$ may be disconnected with connected components $C_{1}, C_{2}, \ldots C_{n}$ as depicted in Fig 1 . The consequence is that after a suitable reordering of rows and columns the Cartan matrix $B$ is block diagonal

$$
B=\operatorname{diag}\left(B_{1}, B_{2}, B_{3} \ldots, B_{n}\right)
$$

where $B_{\beta}$ is the Cartan matrix of the Dynkin diagram for $C_{\beta}$. It is convenient to denote $\Delta_{\beta}=\operatorname{det} B_{\beta}$. Then (3.9) reads

$$
\operatorname{det} A=\Delta_{1} \Delta_{2} \ldots \Delta_{n}\left(2-\sum_{\beta=1}^{n}\left\{\frac{\left.\sum_{i, j \in C_{\beta}} \eta_{i}\left(\operatorname{adj} B_{\beta}\right)_{i j} \eta_{j}\right)}{\Delta_{\beta}}\right\}\right)
$$

This identity provides an efficient tool for evaluating determinants of Cartan matrices iteratively as will be illustrated in the case where the central node is linked to each disjoint component $C_{\beta}$ by a single edge attached to a distinguished node of $C_{\beta}$ that is denoted by *. If $B_{\beta}^{*}$ denotes the Cartan matrix obtained from $B_{\beta}$ by deleting the row and column corresponding to the node $*$ (and is automatically of finite type if $B_{\beta}$ is), and $\Delta_{\beta}^{*}=\operatorname{det} B_{\beta}^{*}$,

$$
\operatorname{det} A=\Delta_{1} \Delta_{2} \ldots \Delta_{n}\left(2-\sum_{\beta=1}^{n} \frac{\Delta_{\beta}^{*}}{\Delta_{\beta}}\right)
$$

Notice that when $C_{n}$ contains no nodes it can be deemed to be an empty diagram so that the reduced diagram $C_{R}$ contains only $n-1$ connected components. The result (3.12) 
ought to reflect this fact and it does so if it is understood that $\Delta_{n}=1$ and $\Delta_{n}^{*}=0$ for an empty diagram.

Let us now use (3.12) to determine a few determinants explicitly. For the case of $s u(N), \operatorname{det} A(s u(N)) \equiv \Delta(s u(N))$ is evaluated by considering the $a_{N-1}=s u(N)$ Dynkin diagram and selecting as the central node one of the two end nodes so that the reduced diagram is connected. Then $\Delta_{1}=\Delta(s u(N-1)), \Delta_{1}^{*}=\Delta(s u(N-2))$ and (3.12) reduces to

$$
\Delta(s u(N))=2 \Delta(s u(N-1))-\Delta(s u(N-2)) .
$$

This is a simple recurrence relation whose general solution is $\Delta(s u(N))=A N+B$. The constants $A$ and $B$ are determined as 1 and 0 respectively by the comments above concerning empty diagrams which imply $\Delta(s u(1))=1$ and $\Delta(s u(0))=0$, yielding the familiar result

$$
\Delta(\operatorname{su}(N))=\operatorname{det} A(\operatorname{su}(N))=N
$$

A similar argument applies to the Dynkin diagram of the Lie algebra $d_{N}=s o(2 N)$ by taking as central node one of the two spinor tips. Again the reduced diagram is connected but this time $\Delta_{1}=\operatorname{det} A(s u(N))=N$ and $\Delta_{1}^{*}=\operatorname{det} A(s u(2)) \operatorname{det} A(s u(N-2))=2(N-2)$ by the results for $s u(N)$. Then (3.12) yields another familiar result

$$
\Delta(s o(2 N))=\operatorname{det} A(s o(2 N))=4 .
$$

More interesting is the Dynkin diagram for $e_{N}$. Selecting as central node the tip of the shortest leg yields $\Delta_{1}=\operatorname{det} A(s u(N))=N$ and $\Delta_{1}^{*}=\operatorname{det} A(\operatorname{su}(3)) \operatorname{det} A(\operatorname{su}(N-3))=$ $3(N-3)$, and so $(3.12)$ gives

$$
\operatorname{det} A\left(e_{N}\right)=9-N
$$

This indicates that $e_{N}$ is Lorentzian if $N \geq 10$, as indeed it is by the preceding discussion. Notice also that $e_{10}$, which is hyperbolic, has a Cartan matrix with determinant -1 so that its root lattice $\Lambda_{R}\left(e_{10}\right)$ is an even, unimodular Lorentzian lattice, a somewhat rare object. In the next section we shall find many more Cartan matrices for such lattices. As explained below $e_{10}$ can be thought of as what is called an overextension of the finite dimensional Lie algebra $e_{8}$. Likewise $e_{11}$ whose Cartan matrix has determinant -2 can be viewed as a very extended version of $e_{8}$. 


\section{When the connected components of the reduced diagram are either finite or affine type}

Suppose that $p$ is the number of connected components of $C_{R}$ that are of affine type. Thus $p$ factors $\Delta_{\beta}=\operatorname{det} B_{\beta}$ vanish, so that, taking account of cancellations it appears from (3.11) that $\operatorname{det} A$ has a $(p-1)$-fold zero. In fact $A$ does have corank $(p-1)$ so that only if $p=1$ is $C$ a Lorentzian diagram. Otherwise it is neither Lorentzian nor affine as its Cartan matrix $A$ has one negative eigenvalue and a $(p-1)$-fold zero eigenvalue.

This is established by displaying a set of simple roots whose scalar products yield the Cartan matrix $A$ whilst spanning a space of dimension $(r-p+1)$ equipped with a Lorentzian scalar product.

First, simple roots are assigned to the reduced diagram, component by component. For each component $C_{\beta}$ assign the simple roots $\alpha_{i}, i \in C_{\beta}$. If $C_{\beta}$ is of finite type these are linearly independent whilst if it is of affine type these are linearly dependent,

$$
\sum_{i \in C_{\beta}} n_{i} \alpha_{i}=0
$$

where the positive integers $n_{i}$ are the Kac labels for the affine diagram $C_{\beta}$. Then the simple roots assigned to the overall diagram $C$ are, in terms of these,

$$
\begin{gathered}
a_{i}=\alpha_{i}+\eta_{i} k=\alpha_{i}-A_{c i} k, \quad i \in C_{R}, \\
a_{c}=-(k+\bar{k}), \quad \text { where } \quad k^{2}=\bar{k}^{2}=0, \quad k \cdot \bar{k}=1 .
\end{gathered}
$$

The vectors $k$ and $\bar{k}$ can be thought to lie in the even self-dual Lorentzian lattice $\mathrm{II}^{1,1}$ whose structure is described in appendix B.

The scalar products of the $a_{i}$ realise the overall Cartan matrix $A$ with $r$ rows and columns yet the roots manifestly span a space of dimension $r-p+1$. Since for each of the $p$ affine components of the reduced diagram $C_{\beta}$

$$
\sum_{i \in C_{\beta}} n_{i} a_{i}=\left(\sum_{i \in C_{\beta}} n_{i} \eta_{i}\right) k
$$

elimination of $k$ yields $p-1$ linear relations amongst these simple roots.

Let us henceforth concentrate on the case that $A$ is Lorentzian so that $p=1$. Let the affine component of the reduced diagram be $C_{1}$ so that $C_{2}, C_{3}, \ldots C_{n}$ are all of finite type. 
Then $\Delta_{1}=\operatorname{det} B_{1}$ vanishes and the right hand side of equation (3.11) simplifies as $n$ of the $(n+1)$ terms vanish, leaving only the $\beta=1$ term in the sum

$$
\operatorname{det} A=-\Delta_{2} \ldots \Delta_{n} \sum_{i, j \in C_{1}} \eta_{i}\left(\operatorname{adj} B_{1}\right)_{i j} \eta_{j} .
$$

Now $C_{1}$ is a connected simply-laced affine diagram and so it has to have the form of an affine Dynkin diagram for a simple, simply-laced affine Kac-Moody algebra $\mathfrak{g}^{(1)}$, say. So $B_{1}=B\left(\mathfrak{g}^{(1)}\right)$. Then its adjugate matrix has the form

$$
\left(\operatorname{adj} B_{1}\right)_{i j}=|\mathrm{Z}(G)| n_{i} n_{j}
$$

where again the integers $n_{i}$ are the Kac labels for $\mathfrak{g}^{(1)}$. They constitute the unique null vector of $B_{1},\left(B_{1}\right)_{i j} n_{j}=0$. But, by definition, its adjugate matrix satisfies $\left(B_{1}\right)_{i j}\left(\operatorname{adj} B_{1}\right)_{j k}=\delta_{i k} \operatorname{det} B_{1}=0$. Hence each column of adj $B_{1}$ is proportional to the null vector. The structure above then follows from the fact that adj $B_{1}$, like $B_{1}$ is symmetric. The normalisation follows by specialising the suffices $i$ and $j$ to the value 0 , denoting the affine node, and remembering that $n_{0}=1$ while $\left(\operatorname{adj} B_{1}\right)_{00}$ is the determinant of the Cartan matrix for $\mathfrak{g}$ and hence equals $|\mathrm{Z}(A(\mathfrak{g}))|$ by $(2.11 \mathrm{~b})$ and comments thereafter.

Hence $\operatorname{det} A$ further simplifies

$$
\operatorname{det} A=-\Delta_{2} \Delta_{3} \ldots \Delta_{n}|\mathrm{Z}(G)|\left(\sum_{i \in C_{1}} n_{i} \eta_{i}\right)^{2},
$$

and $\operatorname{det} A$ is explicitly negative, being expressed as minus a product of positive integers. Notice the remarkable fact that any dependence on the quantities $\eta_{i}=-A_{c i}, i \notin C_{1}$ has disappeared.

The root lattice of any of the diagrams under consideration is an even, integral Lorentzian lattice and, by (2.11) it is self-dual, or self-reciprocal if and only if $\operatorname{det} A$ equals -1 . By (4.6) this is so only if each factor on the right hand side, being an integer, actually equals unity. The only simply-laced connected diagram of finite type with unimodular Cartan matrix is, by the results of the preceding section, the $e_{8}$ Dynkin diagram. So $C_{2}$, $C_{3}, \ldots C_{n}$ must each be of this type. So also must $\mathfrak{g}$ be $e_{8}$ so that $C_{1}$ must be an affine $e_{8}$ diagram, or equivalently, an $e_{9}$ diagram. Finally the factor $\sum_{i \in C_{1}} n_{i} \eta_{i}$ must equal unity. Thus all $\eta_{i}$ here vanish, except for just one that equals unity and must correspond to the node of $C_{1}$ for which the Kac index equals unity. This is the affine node (or one related to it by a diagram symmetry). Thus the central node of $C$ is linked to $C_{1}$ in effect only via the affine node. The dimension of the even, Lorentzian self-dual lattice has therefore 
to be $8 n+2$, in accord with the fact that it is only in these dimensions that such lattices exist. They are denoted $\mathrm{II}^{8 n+1,1}$ and are unique. (For a brief description of these lattices see appendix B.) Nevertheless, notice that, because of the arbitrariness in the quantities $\eta_{i}, i \notin C_{1}$, there are very many Cartan matrices (and therefore many inequivalent KacMoody algebras) that give rise to each of these when $n>1$. If $n=1$ this procedure yields only one Cartan matrix whose root lattice is $\mathrm{II}^{9,1}$ and that is the $e_{10}$ Cartan matrix previously mentioned as an over-extension of the $e_{8}$ Cartan matrix.

The fundamental weights for the overall diagram $C$ are determined in terms of the fundamental weights associated with the reduced diagram as

$$
\begin{gathered}
\ell_{\beta}=\lambda_{\beta}, \quad \beta \in C_{2}, \\
\ell_{c}=-k, \\
\ell_{i}=\lambda_{i}-\frac{n_{i}}{\eta}(k-\bar{k}+\nu), \quad i \in C_{1} .
\end{gathered}
$$

To simplify notation all components of $C_{2}$ of finite type are included in $C_{2}$ which is no longer taken to be connected, and $\lambda_{\beta}$ are the fundamental weights of $C_{2}$. As already mentioned $C_{1}$ has to be the extended Dynkin diagram of a finite dimensional simply-laced simple Lie algebra, $\mathfrak{g}$, or equivalently the Dynkin diagram for the untwisted affine KacMoody algebra $\mathfrak{g}^{(1)} . \lambda_{1}, \lambda_{2}, \ldots \lambda_{r_{1}}$ are the fundamental weights of $\mathfrak{g}$ and $\lambda_{0}=0 . \nu$ records the linkage of the central node to the nodes of $C_{R}$

$$
\nu=\nu(\mathfrak{g})+\nu\left(C_{2}\right), \quad \text { where } \quad \nu(\mathfrak{g})=\sum_{i=1}^{r(\mathfrak{g})} \eta_{i} \lambda_{i} \quad \text { and } \quad \nu\left(C_{2}\right)=\sum_{\beta \in C_{2}} \eta_{\beta} \lambda_{\beta},
$$

and $\eta$ is the quantity that already appeared in the determinant formula (4.6), namely

$$
\eta=\sum_{i \in C_{1}} n_{i} \eta_{i}
$$

Notice that $\eta_{0}=-A_{c 0}$ contributes to $\eta$ but not to $\nu$. It is easy to check that the weights (4.7) do satisfy (2.10), given (4.1) and (4.2).

So the Weyl vector $R$ for the overall diagram $C$, being the sum of the fundamental weights, is

$$
R=\rho\left(C_{2}\right)+\rho(\mathfrak{g})-\frac{h(\mathfrak{g})}{\eta}(k-\bar{k}+\nu)-k,
$$

where $\rho(\mathfrak{g})=\sum_{j=1}^{r(\mathfrak{g})} \lambda_{j}$ is the Weyl vector for $\mathfrak{g}, h(\mathfrak{g})=\sum_{j=0}^{r(\mathfrak{g})} n_{j}$ is the Coxeter number of $\mathfrak{g}$ and $\rho\left(C_{2}\right)$ is the Weyl vector for $C_{2}$. Notice immediately that

$$
R . \ell_{c}=-\frac{h(\mathfrak{g})}{\eta}<0 .
$$


This is very similar to what happened in the previous section, equation (3.5), and means that if there is a principal three dimensional subalgebra it must be so $(1,2)$ rather than so(3). Also

$$
R^{2}=\left[\rho\left(C_{2}\right)-\frac{h(\mathfrak{g})}{\eta} \nu\left(C_{2}\right)\right]^{2}+\left[\rho(\mathfrak{g})-\frac{h(\mathfrak{g})}{\eta} \nu(\mathfrak{g})\right]^{2}-\frac{2 h(\mathfrak{g})(h(\mathfrak{g})+\eta)}{\eta^{2}}
$$

The only negative term is the last and it depends on $C_{1}$ and its linkage to the central node and not at all on $C_{2}$.

Let us consider in turn two possibilities for the linkage between the central node and the affine component $C_{1}$. First suppose that the only link is to the affine node so $\eta_{i}=\delta_{i 0}, i \in C_{1}$. Then $\eta=1$ and $\nu(\mathfrak{g})$ vanishes so that (4.12) reduces to

$$
R^{2}=\rho(\mathfrak{g})^{2}-2 h(\mathfrak{g})(h(\mathfrak{g})+1)+\left[\rho\left(C_{2}\right)-h(\mathfrak{g}) \nu\left(C_{2}\right)\right]^{2}
$$

This can be simplified by the Freudenthal-de Vries strange formula applied to $\mathfrak{g}$,

$$
\rho(\mathfrak{g})^{2}=\frac{h(\mathfrak{g})(h(\mathfrak{g})+1) r(\mathfrak{g})}{12}
$$

to yield

$$
R^{2}=\frac{h(\mathfrak{g})(h(\mathfrak{g})+1)(r(\mathfrak{g})-24)}{12}+\left[\rho\left(C_{2}\right)-h(\mathfrak{g}) \nu\left(C_{2}\right)\right]^{2}
$$

This cannot be negative unless $\mathfrak{g}$ has rank $r(\mathfrak{g})$ less than 24 . Since this is another necessary condition for the presence of a principal so(1,2) subalgebra, it means that there is only a finite number of possibilities for $\mathfrak{g}$ in this situation. There are also constraints on $C_{2}$, as will be discussed below in section 4.1 .

A particularly interesting case is when $C_{2}$ is empty and the Lorentzian algebra with Dynkin diagram $C$ is said to be an "overextension" of $\mathfrak{g}$. Then the condition $R^{2}<0$ reduces to $r(\mathfrak{g})<24$, as noted some time ago [1]. Thus (4.15) can be regarded as a generalisation of this result.

Consider now the remaining possibilities for a single link between the central node and the affine diagram $C_{1}$ so $\eta_{i}=\delta_{i *}$ where $i, * \in C_{1}$ and the Kac label of the node $*$ is $n_{*} \geq 2$. As all nodes of the affine $s u(N)$ diagrams have unit Kac label the only possibilities for the finite dimensional Lie algebra $\mathfrak{g}$ are $e_{6}, e_{7}, e_{8}$ and $d_{n}$. For any choice of node in $e_{6}, e_{7}$ and $e_{8}$ a calculation reveals that the sum of the last two terms in (4.12) is negative so that the squared length of the overall Weyl vector could be negative for some choices of $C_{2}$. If $\mathfrak{g}$ is $d_{n}$ the only possibility is $n_{*}=2$. Then deletion of node $*$ from $C_{1}$ which is the 
affine $d_{n}$ diagram yields a Dynkin diagram of type $d_{p} \oplus d_{q}$, where $p+q=n$ and $p, q \geq 2$. The sum of the last two terms of (4.12) is

$$
\frac{(n-1)}{12}\left(n(n-26)+3(p-q)^{2}\right)
$$

This is negative for a finite number of choices for $p, q$ all entailing $n=p+q<26$.

The conclusion is that whenever there is a single link between the central node and $C_{1}$, the number of possibilities for the affine diagram $C_{1}$ and its linked node is finite when (4.12) is negative. It will be shown below that likewise the number of possibilities for $C_{2}$ is also finite.

Now we look at the choice of the $\eta_{j}$ that seems most likely to produce a principal so(1,2) by minimising $R^{2},(4.12)$, given $\mathfrak{g}$. First force the middle term on the right hand side of $(4.12)$ to vanish by taking $\eta \rho(\mathfrak{g})=h(\mathfrak{g}) \nu(\mathfrak{g})$. The necessary and sufficient condition for this is that all $\eta_{j}, j \in C_{1}$ be equal, to $\eta_{0}$, say. The first term then vanishes if and only if $\eta_{j}, j \in C_{2}$ all equal $\eta_{0}$. In this case

$$
R . \ell_{\beta}=0, \quad \beta \in C_{2}, \quad R . \ell_{c}=-\frac{1}{\eta_{0}}, \text { and } R . \ell_{j}=-\frac{n_{j}}{h(\mathfrak{g}) \eta_{0}}\left(1+\frac{2}{\eta_{0}}\right)<0 .
$$

So $R . \ell_{i}<0, j \in C_{R}$ only if $C_{2}$ is empty. In that case the Lorentzian algebra corresponding to $C$ always has a principal $\mathbf{s o}(1,2)$ subalgebra, whatever $\mathfrak{g}$.

\subsection{Constraints on $C_{2}$}

As we have seen above, if there is a single link between the central node and $C_{1}$, the number of possibilities for the affine diagram $C_{1}$ and its linked node is finite if (4.12) is negative. We want to show now that for each of the finitely many choices for $C_{1}$, there are only finitely many choices for $C_{2}$ that make (4.12) negative. In particular, this shows that within this class of algebras, the rank of the algebras that possess a principal so(1,2) subalgebra is bounded from above.

Given $\mathfrak{g}$, the condition that $(4.12)$ is negative is simply that

$$
\left[\rho\left(C_{2}\right)-h_{0} \nu\left(C_{2}\right)\right]^{2} \leq M_{0}(\mathfrak{g})
$$

where $h_{0}=\frac{h(\mathfrak{g})}{\eta}$, and $M_{0}(\mathfrak{g})=\frac{2 h(\mathfrak{g})(h(\mathfrak{g})+\eta)}{\eta^{2}}-\left(\rho(\mathfrak{g})-h_{0} \nu(\mathfrak{g})\right)^{2}$ only depend on $\mathfrak{g}$. By considering the different possibilities for the algebra $\mathfrak{g}$ it is easy to see that $h_{0} \geq 2$ for each simply-laced Lie algebra.

If $C_{2}$ is not connected, we can split the right hand side of (4.18) into a sum over the simple components. For each simple component, the left hand side of (4.18) is strictly 
positive since $h_{0} \geq 2$. This suffices to show that we can only have finitely many simple components in $C_{2}$. It therefore remains to show that the rank of each simple component must be bounded. This will be done separately for $a_{r}$ and $d_{r}$. In the following we shall write $\rho=\rho\left(C_{2}\right), \nu=\nu\left(C_{2}\right)$.

\section{The case of $a_{r}$}

Let us write the vector $\nu$ in the orthogonal basis of appendix $\mathrm{C}$, i.e.

$$
\nu=\sum_{j=1}^{r+1} l_{j} e_{j}
$$

where $l_{j}$ depends on $\nu$. Given the formula for the fundamental weights (C.7), it now follows that $l_{j+1}-l_{j}=-\eta_{j}$. Thus if we write $\left(\rho-h_{0} \nu\right)$ in the same basis,

$$
\left(\rho-h_{0} \nu\right)=\sum_{j=1}^{r+1} m_{j} e_{j}
$$

then $m_{j+1}-m_{j}=\eta_{j} h_{0}-1$. Since $h_{0} \geq 2$ and $\eta_{j} \in \mathbb{N}_{0}$, at least every other $m_{j}$ is in modulus bigger or equal to $1 / 2$, and therefore

$$
\left(\rho-h_{0} \nu\right)^{2} \geq \frac{r}{8}
$$

Because of (4.18) it is then immediate that the rank of $C_{2}$ must be bounded. It is also obvious from the above argument that only finitely many choices for $\eta_{\beta}, \beta \in C_{2}$ will respect the bound (4.18).

\section{The case of $d_{r}$}

Let us first consider the case when $\nu$ is not a spinor weight. Then, given the formula for the fundamental weights (C.13) and the Weyl vector (C.14) it follows that

$$
\left(\rho-h_{0} \nu\right)=\sum_{i=1}^{r}\left(r-i-h_{0} l_{i}\right) e_{i},
$$

where $l_{i} \in \mathbb{Z}$ depends on $\nu$. Each of the coefficients of $e_{i}$ for $i=1, \ldots, r$ is integer, and since only every $h_{0}^{\text {th }}$ number is divisible by $h_{0}$, at most $\frac{r}{h_{0}}+1$ of them vanish. Thus it follows that

$$
\left(\rho-h_{0} \nu\right)^{2} \geq r\left(1-\frac{1}{h_{0}}\right)-1 .
$$

Since $h_{0} \geq 2$, it then follows that the rank of $C_{2}$ must be bounded.

If $\nu$ is a spinor weight, then each $l_{i}$ is half-odd-integer. Then the same argument applies, except that $h_{0}$ is replaced by $h_{0} / 2$ if $h_{0}$ is even. For all simply-laced algebras 
other than $\mathfrak{g}_{0}=\mathrm{su}(2) h_{0} \geq 3$, and (4.23) is then still sufficient. In the case of $\mathfrak{g}_{0}=\operatorname{su}(2)$, $h_{0} l_{i}$ is then an odd integer, and therefore only every second coefficient in (4.22) can vanish. This is again sufficient to conclude that the rank of $C_{2}$ must be bounded.

The above arguments are somewhat abstract, and it may therefore be instructive to get a better feeling for what the actual bounds are. For the case where the central node is only linked to the affine node of $C_{1}$ and to only one node of each connected component of $C_{2}$, we have made a more detailed analysis (that is sketched in appendix $\mathrm{D}$ ). Within this class of constructions, it is shown there that the rank of an algebra with a principal so(1,2) subalgebra is always less than 42. Actually, this bound is probably not attained, and it would be interesting to find the actual bound. The largest rank example (within this class of constructions) that we have managed to construct has rank 19 and is found by taking $\mathfrak{g}=d_{10}$ and $\mathfrak{g}_{2}=e_{7}$. Its Dynkin diagram is given below.

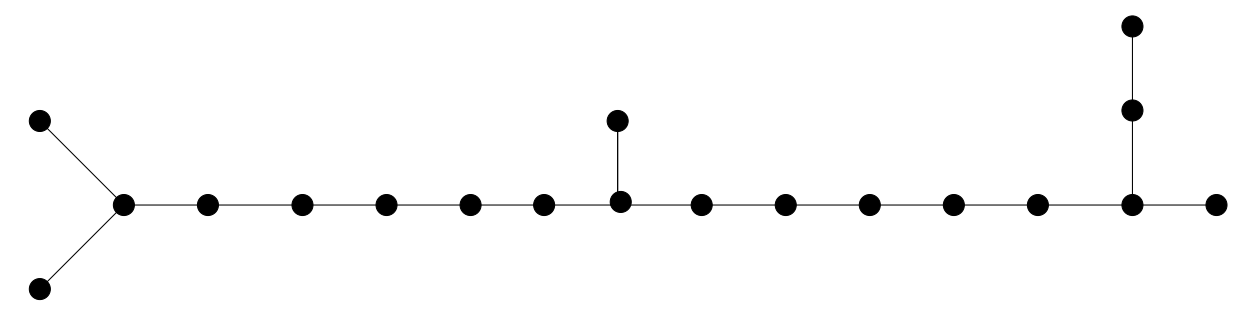

Fig. 3: The Dynkin diagram of the Kac-Moody algebra $\mathfrak{g}$ obtained by taking $\mathfrak{g}=d_{10}$ and $\mathfrak{g}_{2}=e_{7}$.

\section{Very extended Lie algebras}

The Lorentzian Kac-Moody algebras that actually appear in string theory are rather special examples of the algebras we discussed in section 4: they arise by joining an affine Kac-Moody algebra $\mathfrak{g}^{(1)}$ via the affine node to a central node that links in turn to the single finite dimensional Lie algebra $\mathfrak{g}_{2}=\mathrm{su}(2)$. This special construction is a generalisation of the 'over-extension' construction that is explained in [1], and we shall therefore call the resulting Lie algebra 'very extended'. Since these are the examples of primary interest, it may be worthwhile to describe their structure in some detail. We shall also not assume in the following that $\mathfrak{g}$ has a symmetric Cartan matrix.

Let us begin by considering a finite dimensional semi-simple Lie algebra $\mathfrak{g}$ of rank $r$ whose simple roots $\alpha_{i}, i=1, \ldots, r$ span the lattice $\Lambda_{\mathfrak{g}}$. Let us denote the highest root of $\mathfrak{g}$ by $\theta$; we will always normalise the simple roots of $\mathfrak{g}$ such that $\theta^{2}=2$. This can always be done except for the Lie algebra $\mathfrak{g}=g_{2}$ which our analysis does not cover. We choose the convention that the Cartan matrix is defined as $A_{i j}=\frac{2\left(\alpha_{i}, \alpha_{j}\right)}{\left(\alpha_{i}, \alpha_{i}\right)}$. 
In a first step we enlarge the root lattice of $\Lambda_{\mathfrak{g}}$ to be part of

$$
\Lambda_{\mathfrak{g}} \oplus \Pi^{1,1}
$$

by adding to the simple roots of $\mathfrak{g}$ the extended root

$$
\alpha_{0}=k-\theta,
$$

where $k \in \Pi^{1,1} \subset \Lambda_{\mathfrak{g}} \oplus \Pi^{1,1}$ is described in appendix B. The corresponding Lie algebra now has $r+1$ simple roots, and it is just the affine Lie algebra of $\mathfrak{g}$ which is often denoted by $\mathfrak{g}^{(1)}$. However, in view of subsequent developments, we will denote it by $\mathfrak{g}_{0}$. By construction we have $\left(\alpha_{0}, \alpha_{0}\right)=2$ (since $\left.k . k=0\right)$. Let us denote the scalar products involving the new simple root as $\left(\alpha_{0}, \alpha_{i}\right) \equiv q_{i}^{\prime}$ and $2 \frac{\left(\alpha_{i}, \alpha_{0}\right)}{\left(\alpha_{i}, \alpha_{i}\right)} \equiv q_{i}$. The corresponding Cartan matrix then has the form

$$
A_{\mathfrak{g}_{0}}=\left(\begin{array}{ccccc} 
& & & q_{1} \\
& A_{\mathfrak{g}} & & \vdots \\
& & & q_{r} \\
q_{1}^{\prime} & \ldots & q_{r}^{\prime} & 2
\end{array}\right) .
$$

As has been mentioned before, the determinant of the Cartan matrix $A_{\mathfrak{g}_{0}}$ vanishes, $\operatorname{det} A_{\mathfrak{g}_{0}}=0$.

Clearly, the roots of the affine algebra do not span the whole lattice $\Lambda_{\mathfrak{g}} \oplus \Pi^{1,1}$. Rather, the roots of the affine algebra can be characterised as the vectors $x$ in this lattice which are orthogonal to $k$, i.e. $x . k=0$.

We may further extend the affine Lie algebra by adding to the above simple roots yet another simple root namely [1]

$$
\alpha_{-1}=-(k+\bar{k}) \in \Lambda_{\mathfrak{g}} \oplus \Pi^{1,1}
$$

where we have again used the conventions of appendix $\mathrm{B}$. We note that $\alpha_{-1}^{2}=2$, as well as $\left(\alpha_{-1}, \alpha_{0}\right)=-1$ and $\left(\alpha_{-1}, \alpha_{i}\right)=0, i=1 \ldots, r$. The Lie algebra so obtained is called the over-extended Lie algebra, and we shall denote it as $\mathfrak{g}_{-1}$. The Cartan matrix associated to the over-extended Lie algebra has the structure

$$
A_{\mathfrak{g}_{-1}}=\left(\begin{array}{ccccc} 
& & & q_{1} & 0 \\
& A_{\mathfrak{g}} & & \vdots & \vdots \\
& & & q_{r} & 0 \\
q_{1}^{\prime} & \ldots & q_{r}^{\prime} & 2 & -1 \\
0 & \cdots & 0 & -1 & 2
\end{array}\right) .
$$


Examining the form of the Cartan matrix we conclude that

$$
\operatorname{det} A_{\mathfrak{g}_{-1}}=2 \operatorname{det} A_{\mathfrak{g}_{0}}-\operatorname{det} A_{\mathfrak{g}}=-\operatorname{det} A_{\mathfrak{g}} .
$$

Clearly, the root lattice of $\mathfrak{g}_{-1}$ is $\Lambda_{\mathfrak{g}_{-1}}=\Lambda_{\mathfrak{g}} \oplus \Pi^{1,1}$. The algebra $\mathfrak{g}_{-1}$ is therefore Lorentzian. It is possible to enlarge the Lie algebra even further by considering the lattice

$$
\Lambda_{\mathfrak{g}} \oplus \Pi^{1,1} \oplus \Pi^{1,1}=\Lambda_{\mathfrak{g}_{-1}} \oplus \Pi^{1,1} .
$$

We denote the analogue of $k$ and $\bar{k}$ in the second $\Pi^{1,1}$ lattice by $l$ and $\bar{l}$, respectively. We now add the new simple root

$$
\alpha_{-2}=k-(l+\bar{l}) .
$$

We then have that $\left(\alpha_{-2}, \alpha_{-2}\right)=2,\left(\alpha_{-2}, \alpha_{-1}\right)=-1$, while all other scalar products involving $\alpha_{-2}$ vanish. Let us denote the resulting Kac-Moody algebra by $\mathfrak{g}_{-2}$. The corresponding Cartan matrix is then of the form

$$
A_{\mathfrak{g}_{-2}}=\left(\begin{array}{cccccc} 
& & & q_{1} & 0 & 0 \\
& A_{\mathfrak{g}} & & \vdots & \vdots & \vdots \\
& & & q_{r} & 0 & 0 \\
q_{1}^{\prime} & \ldots & q_{r}^{\prime} & 2 & -1 & 0 \\
0 & \cdots & 0 & -1 & 2 & -1 \\
0 & \ldots & 0 & 0 & -1 & 2
\end{array}\right) .
$$

This Cartan matrix is precisely the Cartan matrix that is obtained from the construction in section 4 with $\mathfrak{g}_{2}=\mathrm{su}(2)$. Examining the form of this Cartan matrix we conclude that

$$
\operatorname{det} A_{\mathfrak{g}_{-2}}=2 \operatorname{det} A_{\mathfrak{g}_{-1}}-\operatorname{det} A_{\mathfrak{g}_{0}}=2 \operatorname{det} A_{\mathfrak{g}_{-1}}=-2 \operatorname{det} A_{\mathfrak{g}} .
$$

This is in agreement with (4.6) since the determinant of the Cartan matrix of $\mathrm{su}(2)$ equals 2 and $\eta$, defined in (4.9), equals $\eta=1$. The root lattice of $\mathfrak{g}_{-2}$ consists of all vectors $x$ in $\Lambda_{\mathfrak{g}} \oplus \Pi^{1,1} \oplus \Pi^{1,1}$ which are orthogonal to the time-like vector

$$
s=l-\bar{l}=(1,1),
$$

where we have used the notation of appendix B. This implies, in particular, that $\mathfrak{g}_{-2}$ is a Lorentzian algebra.

As before, it is straightforward to calculate the fundamental weights of the overextended and very extended algebras. In the over-extended case the fundamental weights are given as

$$
\begin{aligned}
\lambda_{i} & =\lambda_{i}^{\mathrm{f}}-\left(\lambda_{i}^{\mathrm{f}}, \theta\right)(k-\bar{k}), \quad i=1, \ldots, r, \\
\lambda_{0} & =-(k-\bar{k}), \\
\lambda_{-1} & =-k
\end{aligned}
$$


where $\lambda_{i}^{\mathrm{f}}$ are the fundamental weights of $\mathfrak{g}$. On the other hand, the fundamental weights of the very extended algebra are

$$
\begin{aligned}
\lambda_{i} & =\lambda_{i}^{\mathrm{f}}-\left(\lambda_{i}^{\mathrm{f}}, \theta\right)\left(k-\bar{k}-\frac{1}{2}(l+\bar{l})\right), \quad i=1, \ldots, r, \\
\lambda_{0} & =-\left(k-\bar{k}-\frac{1}{2}(l+\bar{l})\right), \\
\lambda_{-1} & =-k \\
\lambda_{-2} & =-\frac{1}{2}(l+\bar{l}) .
\end{aligned}
$$

It was shown in [1] that the Weyl vector of an over-extended algebra is given by

$$
\rho=\rho_{\mathrm{f}}+h \bar{k}-(h+1) k,
$$

where $\rho_{\mathrm{f}}$ is the Weyl vector of the underlying finite dimensional Lie algebra $\mathfrak{g}$, and $h$ is its Coxeter number. Similarly, the Weyl vector of the very extended Kac-Moody algebras is given by

$$
\rho=\rho_{\mathrm{f}}+h \bar{k}-(h+1) k-\frac{1}{2}(1-h)(l+\bar{l}) .
$$

\subsection{Weight lattices}

We now construct the weight lattices of the Kac-Moody algebras introduced above. For simplicity we shall only consider the simply-laced case for which the weight lattice $\Lambda_{W}$ is just the dual $\Lambda_{W}=\Lambda_{R}^{*}$ of the root lattice $\Lambda_{R}$. These lattices can be easily found, using the fact that for any two lattices $\Lambda_{1}$ and $\Lambda_{2}$ we have

$$
\left(\Lambda_{1} \oplus \Lambda_{2}\right)^{\star}=\Lambda_{1}^{\star} \oplus \Lambda_{2}^{\star}
$$

Now the lattice $\Pi^{1,1}$ is self-dual, and thus the weight lattice of $\mathfrak{g}_{-1}$ is simply given by

$$
\left(\Lambda_{\mathfrak{g}_{-1}}\right)^{\star}=\Lambda_{\mathfrak{g}}^{\star} \oplus \Pi^{1,1}
$$

In particular it follows that

$$
\frac{\left(\Lambda_{\mathfrak{g}_{-1}}\right)^{\star}}{\left(\Lambda_{\mathfrak{g}_{-1}}\right)}=\mathrm{Z}_{\mathfrak{g}} .
$$

Given (2.11), this result is consistent with the relation between the determinants of equation (5.6).

The weight lattice for $\mathfrak{g}_{-2}$ is given as

$$
\left(\Lambda_{\mathfrak{g}_{-2}}\right)^{\star}=\Lambda_{\mathfrak{g}}^{\star} \oplus \Pi^{1,1} \oplus\{(r,-r): 2 r \in \mathbb{Z}\}
$$


where we have used the conventions of appendix B. In deriving (5.19) we have noted that the root lattice of $\mathfrak{g}_{-2}$ is

$$
\Lambda_{\mathfrak{g}_{-2}}=\Lambda_{\mathfrak{g}} \oplus \Pi^{1,1} \oplus\{(t,-t): t \in \mathbb{Z}\}
$$

since $l+\bar{l}=(1,-1)$. The last lattice in (5.19) is generated by $f=(1 / 2,-1 / 2)$ which is not in $\Pi^{1,1}$, but for which $2 f \in \Pi^{1,1}$. Thus we conclude that

$$
\frac{\left(\Lambda_{\mathfrak{g}_{-2}}\right)^{\star}}{\left(\Lambda_{\mathfrak{g}_{-2}}\right)}=\mathrm{Z}_{\mathfrak{g}} \times \mathbb{Z}_{2} .
$$

Here the $\mathbb{Z}_{2}$ results from the fact that the dual lattice contains the vector $f$ in the last factor of $\Pi^{1,1}$. This is consistent with the factor of 2 between the two determinants of equation (5.10).

\subsection{The relation to self-dual lattices}

The above extensions were carried out for any finite dimensional semi-simple Lie algebra $\mathfrak{g}$ of rank $r$, but we now consider in detail the resulting algebras when $\Lambda_{\mathfrak{g}}$ is an even self-dual lattice of dimension $r$, or a sublattice of such a lattice. Even self-dual Euclidean lattices only exist in dimensions $D=8 n, n=1,2, \ldots$.

The first non-trivial example of such a lattice occurs in eight dimensions where there is only one such lattice, the root lattice of $e_{8}$. Let us denote the corresponding affine, over-extended and very extended algebras by $e_{9}, e_{10}$ and $e_{11}$, respectively. We can choose a basis for the root lattice of $e_{8}, \Lambda_{e_{8}}$, to be

$$
\begin{aligned}
& \alpha_{1}=(0,0,0,0,0,1,-1,0), \\
& \alpha_{2}=(0,0,0,0,1,-1,0,0), \\
& \alpha_{3}=(0,0,0,1,-1,0,0,0), \\
& \alpha_{4}=(0,0,1,-1,0,0,0,0), \\
& \alpha_{5}=(0,1,-1,0,0,0,0,0), \\
& \alpha_{6}=(-1,-1,0,0,0,0,0,0), \\
& \alpha_{7}=\left(\frac{1}{2}, \frac{1}{2}, \frac{1}{2}, \frac{1}{2}, \frac{1}{2}, \frac{1}{2}, \frac{1}{2}, \frac{1}{2}\right), \\
& \alpha_{8}=(1,-1,0,0,0,0,0,0) .
\end{aligned}
$$

In order to describe the extension and over-extension of $e_{8}$ we consider the lattice $\Lambda_{e_{8}} \oplus \Pi^{1,1}$. The affine root that gives $e_{9}$ is now given by

$$
\alpha_{0}=k-\theta=(-\theta ;(1,0)) \text {, }
$$


where $\theta \in \Lambda_{e_{8}}$ is the highest root of $e_{8}$, which, with the above choice of simple roots, is

$$
\theta=(0,0,0,0,0,0,1,-1)
$$

Finally, the over-extended root that enhances this to $e_{10}$ can then be chosen to be

$$
\alpha_{-1}=-(k+\bar{k})=(\mathbf{0} ;(-1,1))
$$

It is easy to see (and in fact well known [1]) that this construction gives the root lattice of $e_{10}$.

The lattice $\Lambda_{e_{8}} \oplus \Pi^{1,1}$ is clearly self-dual by virtue of equation (5.16). It is of Lorentzian signature and even. Such lattices only occur in dimensions $D=8 n+2, n=0,1,2 \ldots$, and the lattice in each dimension is unique and usually denoted by $\Pi^{8 n+1,1}$. It follows that the root lattice of $e_{10}$ is precisely this lattice for $n=1$, i.e. $\Lambda_{e_{10}}=\Pi^{9,1}$.

Finally, we consider the lattice

$$
\Pi^{9,1} \oplus \Pi^{1,1}=\Lambda_{e_{8}} \oplus \Pi^{1,1} \oplus \Pi^{1,1}=\Pi^{10,2}
$$

where the latter lattice is the unique even self-dual lattice of signature $(10,2)$. The very extended root is given by

$$
\alpha_{-2}=k-(l+\bar{l})=(\mathbf{0} ;(1,0) ;(-1,1))
$$

The corresponding algebra, $e_{11}$, has been argued to be a symmetry of M-theory in [4]. From equation (5.21) it now follows that

$$
\frac{\Lambda_{e_{11}}^{\star}}{\Lambda_{e_{11}}}=\mathbb{Z}_{2} .
$$

\subsection{The 24-dimensional case}

Next let us consider the extensions of a finite dimensional semi-simple Lie algebra of rank 24 whose root lattice is a sublattice of an even self-dual Euclidean lattice in dimension 24. In dimension 24 , there are 24 such lattices, the so-called Niemeier lattices [12]. One of the Niemeier lattices contains the root lattice of $d_{24}$, that can be taken to be spanned by the vectors in $\mathbb{Z}^{24}$ of the form

$$
\alpha_{i}=\left(0^{i-1}, 1,-1,0^{23-i}\right), \quad i=1, \ldots, 23, \quad \alpha_{24}=\left(0^{22}, 1,1\right)
$$

The root lattice of $d_{24}$ is not self-dual by itself since

$$
\frac{\Lambda_{d_{24}}^{\star}}{\Lambda_{d_{24}}}=\mathbb{Z}_{2} \times \mathbb{Z}_{2},
$$


which is consistent with the fact that $\operatorname{det} A_{d_{24}}=4$. The corresponding self-dual lattice is given by

$$
\Lambda_{d_{24}}^{N}=\frac{\Lambda_{d_{24}}^{\star}}{\mathbb{Z}_{2}}
$$

It is obtained from the root lattice of $d_{24}, \Lambda_{d_{24}}$, by adjoining a point of length squared six,

$$
g=\left[\left(\frac{1}{2}\right)^{24}\right]
$$

It is easy to see that $g \in \Lambda_{d_{24}}^{\star}$, and that $2 g \in \Lambda_{d_{24}}$.

Let us denote by $k_{26}$ the over-extension of $d_{24}$ that is obtained by adding to $d_{24}$ the affine and over-extended roots. The rank of $k_{26}$ is 26 , and its root lattice is

$$
\Lambda_{k_{26}}=\Lambda_{d_{24}} \oplus \Pi^{1,1}
$$

It is spanned by the roots of equation (5.29), together with

$$
\begin{aligned}
\alpha_{0} & =\left(\left(-1,-1,0^{22}\right) ;(1,0)\right), \\
\alpha_{-1} & =\left(\left(0^{24}\right) ;(-1,1)\right) .
\end{aligned}
$$

We have

$$
\frac{\Lambda_{k_{26}}^{*}}{\mathbb{Z}_{2}}=\frac{\Lambda_{d_{24}}^{*}}{\mathbb{Z}_{2}} \oplus \Pi^{1,1}=\Lambda_{d_{24}}^{N} \oplus \Pi^{1,1}=\Pi^{25,1},
$$

since the lattice $\frac{\Lambda_{d_{24}}^{*}}{\mathbb{Z}_{2}} \oplus \Pi^{1,1}$ is an even self-dual lattice of dimensional 26. It thus follows that $\Lambda_{k_{26}}$ is contained in $\Pi^{25,1}$.

Finally we consider the further extension of $d_{24}$ by considering the lattice

$$
\Lambda_{d_{24}} \oplus \Pi^{1,1} \oplus \Pi^{1,1}
$$

and adding the simple root

$$
\alpha_{-2}=\left(\left(0^{24}\right) ;(1,0) ;(-1,1)\right)
$$

as discussed for the general case above. We shall denote the corresponding algebra by $k_{27}$; it has been argued to be a symmetry of the 26-dimensional closed bosonic string [4]. It also follows that

$$
\frac{\Lambda_{k_{27}}^{\star}}{\Lambda_{k_{27}}}=\mathbb{Z}_{2} \times \mathbb{Z}_{2} \times \mathbb{Z}_{2}
$$




\subsection{The 16-dimensional case}

For completeness, let us conclude this section with a discussion of the very extended Lie algebra associated to the rank 16 algebra $d_{16}$. As in the previous section, the corresponding root lattice is not self-dual since

$$
\frac{\Lambda_{d_{16}}^{\star}}{\Lambda_{d_{16}}}=\mathbb{Z}_{2} \times \mathbb{Z}_{2} .
$$

The corresponding self-dual lattice is given by

$$
\Lambda_{d_{16}}^{s}=\frac{\Lambda_{d_{16}}^{\star}}{\mathbb{Z}_{2}} .
$$

It is obtained from the root lattice of $d_{16}, \Lambda_{d_{16}}$, by adjoining a point of length squared four,

$$
g=\left[\left(\frac{1}{2}\right)^{16}\right] .
$$

Let us denote by $m_{18}$ the over-extension of $d_{16}$ that is obtained by adding to $d_{16}$ the affine and over-extended roots. The rank of $m_{18}$ is 18 , and its root lattice is

$$
\Lambda_{m_{18}}=\Lambda_{d_{16}} \oplus \Pi^{1,1} .
$$

In particular, we therefore have

$$
\frac{\Lambda_{m_{18}}^{*}}{\mathbb{Z}_{2}}=\frac{\Lambda_{d_{16}}^{*}}{\mathbb{Z}_{2}} \oplus \Pi^{1,1}=\Lambda_{d_{16}}^{s} \oplus \Pi^{1,1}=\Pi^{17,1},
$$

and thus $\Lambda_{m_{18}}$ is contained in $\Pi^{17,1}$.

Finally we consider the further extension of $d_{16}$ by considering the lattice

$$
\Lambda_{d_{16}} \oplus \Pi^{1,1} \oplus \Pi^{1,1}
$$

and adding the simple root

$$
\alpha_{-2}=\left(\left(0^{16}\right) ;(1,0) ;(-1,1)\right),
$$

as discussed for the general case above. We shall denote the corresponding algebra by $m_{19}$.

We observe that constructing the very extended Lorentzian Kac-Moody algebras based on Euclidean self-dual lattices leads to a very specific set of algebras, namely $e_{11}, m_{19}$ and $k_{27}$. Remakably, $e_{11}$ and $k_{27}$ are thought to be symmetries of $\mathrm{M}$ theory and the 26-dimensional bosonic string [4]. The above construction also makes it clear that the symmetries of these two theories are related to the unique even self-dual Lorentzian lattices in ten and 26 dimensions, respectively. These observations encourage the speculation that there should also exist a 18 -dimensional string with a symmetry $k_{19}$. These and other implications for string theory will be discussed elsewhere. 


\subsection{Principal so(1,2) subalgebras}

It may also be interesting to analyse which of the over-extended and very extended Kac Moody algebras possess a principal so( 1,2$)$ subalgebra. As before we have to analyse the condition of equation (A.8). Using (A.9), as well as the explicit expressions for the fundamental weights given in (5.12) and (5.13), we find that for an over-extended KacMoody algebra the left hand side of equation (A.8) is

$$
\begin{aligned}
\sum_{a} A_{a-1}^{-1} & =-h \\
\sum_{a} A_{a 0}^{-1} & =-(2 h+1), \\
\sum_{a} A_{a j}^{-1} & =-\frac{1}{2} n_{j}\left(\alpha_{j}, \alpha_{j}\right)(2 h+1)+\sum_{i} A_{\mathrm{f} i j}^{-1} .
\end{aligned}
$$

Here $h, n_{i}, A_{\mathrm{f} i j}^{-1}$ are the Coxeter number, the Kac labels, and the inverse Cartan matrix of the finite dimensional Lie algebra $\mathfrak{g}$, respectively.

Similarly, we find for the case of the very extended Kac-Moody algebra

$$
\begin{aligned}
\sum_{a} A_{a-2}^{-1} & =-\frac{1}{2}(h-1), \\
\sum_{a} A_{a-1}^{-1} & =-h \\
\sum_{a} A_{a 0}^{-1} & =-\frac{3}{2}(h+1), \\
\sum_{a} A_{a j}^{-1} & =-\frac{3}{4} n_{j}\left(\alpha_{j}, \alpha_{j}\right)(h+1)+\sum_{i} A_{\mathrm{f} i j}^{-1} .
\end{aligned}
$$

We observe that in both cases only the sums $\sum_{a} A_{a j}^{-1}$ do not automatically satisfy the required condition of equation (A.8). The relevant condition depends therefore on the Kac labels, Coexter numbers, and the corresponding sums in the finite dimensional Lie algebra. For the case of the classical Lie algebras, the relevant data have been collected in appendix C.

As an example, let us consider the case of the very extended $a_{n}$ algebra in more detail. Using equation (C.9) of appendix $\mathrm{C}$ we find that

$$
\sum_{a} A_{a j}^{-1}=-\frac{3}{2}(n+2)+\frac{j}{2}(n+1-j) .
$$

This has its maximum when $j=\frac{n+1}{2}$ for $n$ odd and $j=\frac{n}{2}$ for $n$ even. In the first case the maximum of $\sum_{a} A_{a j}^{-1}$ is $\frac{1}{8}\left(n^{2}-10 n-23\right)$ while in the latter case it is $\frac{1}{8}\left(n^{2}-10 n-24\right)$. 
Hence $\sum_{a} A_{a j}^{-1}$ is non-positive if and only if $n \leq 12$, and thus a principal so(1,2) subalgebra exists for the very extended $a_{n}$ algebra if $n \leq 12$. We summarise the results for all overand very extended Lie algebras in the following table.

\begin{tabular}{|l|l|l|}
\hline & over-extended & very extended \\
\hline$a_{n}$ & $n \leq 16$ & $n \leq 12$ \\
$d_{n}$ & $n \leq 16$ & $n \leq 12$ \\
$e_{n}$ & $n=6,7,8$ & $n=6,7,8$ \\
$b_{n}$ & $n \leq 15$ & $n \leq 11$ \\
$c_{n}$ & $n \leq 8$ & $n \leq 6$ \\
$f_{4}$ & yes & yes \\
$g_{2}$ & yes & yes \\
\hline
\end{tabular}

Table 1: The algebras with principal so(1,2) subalgebras.

The algebras of particular interest to string theory are $e_{11}$ and $k_{27}$. These algebras are the very extended algebras corresponding to $e_{8}$ and $d_{24}$, respectively. The above table implies that while $e_{11}$ admits a principal so(1,2) subalgebra, $k_{27}$ does not. We also note that the other algebra that is related to self-dual lattices, $m_{19}$ (see section 5.4 ), also does not admit a principal so $(1,2)$ subalgebra since it is the very extended algebra corresponding to $d_{16}$.

\section{Other constructions}

Up to now we have discussed Lorentzian Kac-Moody algebras that arise by means of a certain simple construction. While these Kac-Moody algebras may be preferred in some way, it is clear that they do not account for all Lorentzian Kac-Moody algebras, and not even for all those with a principal so(1,2) subalgebra.

In this section we want to describe some other classes of Lorentzian Kac-Moody algebras that can be obtained by similar types of constructions. In each case we shall also analyse for which examples the resulting algebra has a principal so(1,2) subalgebra.

\subsection{Adding a different node}

The simplest modification of the above construction leading to a very extended Lie algebra is to attach the very extended node at a different place in the Dynkin diagram of the over-extended algebra. As before, we shall take the roots to belong to the lattice

$$
\Lambda_{\mathfrak{g}} \oplus \Pi^{1,1} \oplus \Pi^{1,1}=\Lambda_{\mathfrak{g}_{-1}} \oplus \Pi^{1,1}
$$


We take the roots of our new algebra to be the roots of the over-extended algebra (see section 5), except that we replace $\alpha_{i_{0}}$ by $\widehat{\alpha}_{i_{0}}=\alpha_{i_{0}}+l$, where $l$ is defined as in section 5 and $i_{0}$ is a chosen index on the Dynkin diagram; in addition we choose $\alpha_{-2}=-(l+\bar{l})$. The corresponding Dynkin diagram is then the diagram that is obtained from the Dynkin diagram of the over-extended algebra by adding a node that is attached to the $i_{0}^{\text {th }}$ node. The roots of this new algebra are orthogonal to the vector

$$
s=(l-\bar{l})+2 \frac{\lambda_{i_{0}}}{\left(\alpha_{i_{0}}, \alpha_{i_{0}}\right)}+2 \frac{\left(\lambda_{i_{0}}, \theta\right)}{\left(\alpha_{i_{0}}, \alpha_{i_{0}}\right)}(\bar{k}-k) .
$$

The resulting algebra is therefore Lorentzian if $s$ is time-like, i.e. if

$$
4 \frac{\left(\lambda_{i_{0}}, \lambda_{i_{0}}\right)}{\left(\alpha_{i_{0}}, \alpha_{i_{0}}\right)^{2}}<2\left(1+n_{i_{0}}^{2}\right)
$$

For example, if we take $\mathfrak{g}=a_{n}$, then the algebra is Lorentzian if

$$
i_{0}\left(n+1-i_{0}\right)<4(n+1) .
$$

We have also analysed (using Maple) which of these algebras have a principal so(1,2) subalgebra. We have found that for $n=16$, two of the algebras so obtained have a principal so(1,2) subalgebra; their Dynkin diagrams are shown below.
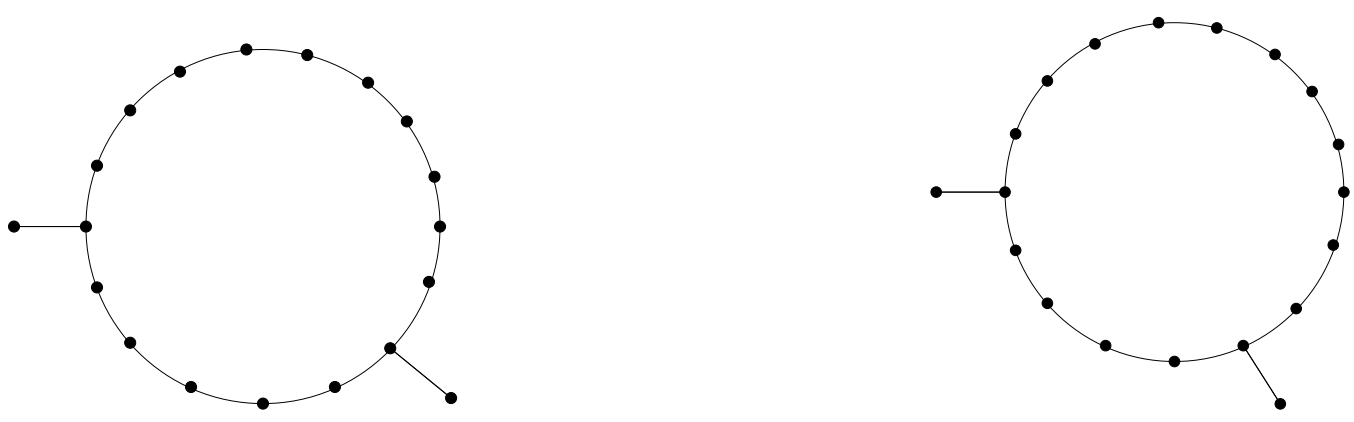

Fig. 4: Dynkin diagrams of Lorentzian Kac-Moody algebras with a principal so(1,2) subalgebra that cannot be obtained by any of the constructions described in section 3 and 4.

\subsection{Symmetric fusion}

The construction of section 4 is somewhat asymmetric in that one affine Kac-Moody algebra is singled out. In this section we want to describe a more symmetrical construction that also gives rise to a Lorentzian Kac-Moody algebra. As will become apparent, this construction can actually be regarded as a special case of the construction of section 4 . However, it may nevertheless be interesting to discuss it in its own right. 
Suppose $\mathfrak{g}_{1}$ and $\mathfrak{g}_{2}$ are two finite dimensional simply-laced simple Lie algebras of rank $r_{1}$ and $r_{2}$, respectively. We want to construct a Lorentzian algebra $\mathfrak{g}_{1} \diamond \mathfrak{g}_{2}$ whose rank is $r_{1}+r_{2}+2$. The root lattice of this algebra will be given by

$$
\Lambda_{\mathfrak{g}_{1}} \oplus \Pi^{1,1} \oplus \Lambda_{\mathfrak{g}_{2}} .
$$

We take the simple roots of the algebra $\mathfrak{g}_{1} \diamond \mathfrak{g}_{2}$ to be those of $\mathfrak{g}_{1}$ and $\mathfrak{g}_{2}$, which we denote by $\alpha_{i}, i=1, \ldots, r_{1}$ and $\beta_{j}, j=1, \ldots, r_{2}$, respectively. We add to these two further simple roots,

$$
\alpha_{0}=k-\theta_{1} \quad \text { and } \quad \beta_{0}=-\bar{k}-\theta_{2},
$$

where $k$ and $\bar{k}$ belong to $\Pi^{1,1}$, as explained in appendix $\mathrm{B}$, and $\theta_{1}$ and $\theta_{2}$ are the highest roots of $\mathfrak{g}_{1}$ and $\mathfrak{g}_{2}$, respectively. Since $\mathfrak{g}_{1}$ and $\mathfrak{g}_{2}$ are simply-laced algebras, we have $\alpha_{0}^{2}=\beta_{0}^{2}=2,\left(\alpha_{0}, \beta_{0}\right)=-1$. The corresponding Cartan matrix is then given by

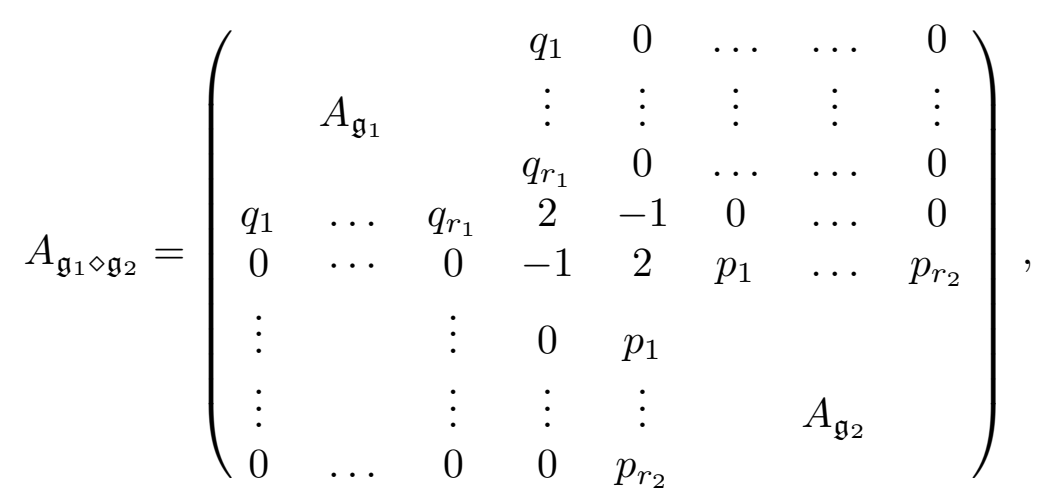

where $q_{i}=\left(\alpha_{i}, \alpha_{0}\right)$, and $p_{j}=\left(\beta_{j}, \beta_{0}\right)$. By construction, it is clear that the Dynkin diagram of $\mathfrak{g}_{1} \diamond \mathfrak{g}_{2}$ contains the Dynkin diagrams of $\mathfrak{g}_{1}^{(1)}$ and $\mathfrak{g}_{2}^{(1)}$, respectively. In fact, it is obtained by joining the two affine diagrams with a single line between the two affine roots. If we think of the affine node of either $\mathfrak{g}_{1}^{(1)}$ or $\mathfrak{g}_{2}^{(1)}$ as the central node, the Dynkin diagram is then of the form described in section 4.

By considering the column associated with the root $\alpha_{0}$ we can calculate the determinant of the corresponding Cartan matrix, and we find as before that

$$
\operatorname{det} A_{\mathfrak{g}_{1} \diamond \mathfrak{g}_{2}}=-\operatorname{det} A_{\mathfrak{g}_{1}} \operatorname{det} A_{\mathfrak{g}_{2}} .
$$

This is in agreement with (4.6). It also follows from the analysis of section 4 that the Kac-Moody algebra $\mathfrak{g}_{1} \diamond \mathfrak{g}_{2}$ is Lorentzian.

Next we want to analyse for which cases this Lorentzian algebra has a principal so $(1,2)$ subalgebra. As before, we can determine the weights, and we find that they are given by

$$
\begin{array}{ll}
\ell_{i}^{(1)}=\lambda_{i}^{(1)}+\left(\lambda_{i}^{(1)}, \theta_{1}\right) \bar{k}, & \ell_{\alpha_{0}}=\bar{k}, \\
\ell_{i}^{(2)}=\lambda_{i}^{(2)}-\left(\lambda_{i}^{(2)}, \theta_{2}\right) k, & \ell_{\beta_{0}}=-k,
\end{array}
$$


where $\lambda_{i}^{(p)}$ are the fundamental weights of $\mathfrak{g}_{p}, p=1,2$. It is straightforward to show that

$$
\sum_{a}\left(A_{\mathfrak{g}_{1} \diamond \mathfrak{g}_{2}}\right)_{a}^{-1} \alpha_{0}=-h\left(\mathfrak{g}_{2}\right)<0, \quad \sum_{a}\left(A_{\mathfrak{g}_{1} \diamond \mathfrak{g}_{2}}\right)_{a j}^{-1}=-n_{j}^{(1)} h\left(\mathfrak{g}_{2}\right)+\sum_{i=1}^{r_{1}} A_{\mathfrak{g}_{1} i j}^{-1}
$$

where $j$ denotes a node of $\mathfrak{g}_{1}$. The sums over the other columns may be obtained from the above by exchanging $1 \leftrightarrow 2$. As in our discussion of section 5.5 we therefore conclude that a principal so(1,2) subalgebra exists if the second sum in equation (6.10) is also negative. Let us discuss a few examples in detail.

For the case of $\mathfrak{g}_{1}=a_{n_{1}}, \mathfrak{g}_{2}=a_{n_{2}}$ we find, using the results of appendix $\mathrm{C}$, that there is a principal so(1,2) subalgebra if $\left(n_{1}+1\right)^{2} \leq 8\left(n_{2}+1\right)$ and $\left(n_{2}+1\right)^{2} \leq 8\left(n_{1}+1\right)$. (In deriving these inequalities we have assumed that both $n_{1}$ and $n_{2}$ are odd, but similar inequalities also hold if $n_{1}$ or $n_{2}$ are even.) By squaring the first condition and using the second we conclude that $n_{1} \leq 7, n_{2} \leq 7$. It is straightforward to find the solutions (taking, without loss of generality, $n_{2} \geq n_{1}$ ): apart from the case $n_{1}=n_{2}=1, \ldots, 7$ and $n_{2}=n_{1}+1=1, \ldots, 5$ there is the one additional solution $n_{2}=3, n_{1}=1$.

Similarly we find that for the case of $\mathfrak{g}_{1}=d_{n_{1}}, \mathfrak{g}_{2}=d_{n_{2}}$ there exists a principal $\mathrm{so}(1,2)$ subalgebra if $n_{1}=n_{2}=3, \ldots, 8, n_{2}=n_{1}+1, n_{1}=3, \ldots, 7$ and $n_{2}=n_{1}+2=4,5,6$. Also, for the case of $\mathfrak{g}_{1}=a_{n_{1}}, \mathfrak{g}_{2}=d_{n_{2}}$ we find that there exists a principal $\operatorname{so}(1,2)$ subalgebra only if $n_{1} \leq 7$ and $n_{2} \leq 6$.

Finally, we have checked that the algebras $a_{n} \diamond e_{m}$ only have a principal so(1,2) subalgebra provided that $m=6$ and $n=7,8$, and that the algebras $d_{n} \diamond e_{m}$ only have a principal so(1,2) subalgebra provided that $m=6, n=5,6,7$ or $m=7, n=8,9$. Furthermore the algebras $e_{n} \diamond e_{m}$ have a principal so $(1,2)$ subalgebra if and only if $n=m$ with $n=6,7,8$.

It follows from (6.5) and (6.8) that the symmetric fusion of two finite dimensional simple Lie algebras gives rise to an even self-dual root lattices provided that the two finite dimensional Lie algebras are separately self-dual. The only example is therefore the rank 18 Lorentzian algebra $e_{8} \diamond e_{8}$. Another interesting example is the algebra $e_{8} \diamond d_{16}$, that actually equals the algebra $k_{26}$ discussed earlier. The root lattice of this algebra is not self-dual, but as explained in section 5, can be made self-dual by the addition of a spinor weight of $d_{16}$. 


\section{Conclusions}

In this paper we have described and analysed a certain subclass of (Lorentzian) KacMoody algebras that are in many respects rather amenable to a general analysis. These algebras are characterised by the property that their Dynkin diagram contains at least one node, upon whose deletion the diagram becomes that of a direct sum of affine and finite Lie algebras. We have described the conditions under which these algebras are actually Lorentzian, and we have given explicit descriptions for their simple roots and fundamental weights. We have also found simple formulae for the determinants of the corresponding Cartan matrices. Using similar techniques one can derive their characteristic polynomials, thus reproducing (for the case of the finite Lie algebras) known results in a rather elegant fashion.

We have discussed the Lorentzian algebras whose root lattices are self-dual. In particular, we have shown how to construct, for a given even self-dual Lorentzian lattice, a large number of inequivalent algebras whose root lattice is the given Lorentzian lattice. Finally we have studied the question of whether our Lie algebras possess a principal so(1,2) subalgebra.

A special subclass of the algebras we have considered are what we called very extended Lie algebras. These very extended algebras arise as symmetries of M-theory and the bosonic string [4], thus suggesting that the subclass of algebras described in this paper may play an important rôle in physics.

The methods we have described in this paper will probably generalise to other classes of algebras. In particular, one can use for example our determinant formulae iteratively to analyse Dynkin diagrams that reduce to that of affine and finite Lie algebras upon deletion of two or mode nodes, etc. It would be interesting to explore these ideas further.

\section{Acknowledgements}

We thank Hermann Nicolai and Andrew Pressley for useful conversations. MRG is supported by the Royal Society via a University Research Fellowship. He is grateful to the Isaac Newton Institute for hospitality while this paper was being completed. DIO and PCW are grateful to CERN for hospitality during an early stage of this work. MRG, DIO and PCW acknowledge partial support from the EU network 'Superstrings' (HPRN-CT2000-00122), and MRG and PCW acknowledge partial support from the PPARC special grant 'String Theory and Realistic Field Theory', PPA/G/S/1998/0061. 


\section{Appendix A. Principal so(1,2) subalgebras}

It is well known that every finite dimensional semi-simple Lie algebra contains a principal so(3) subalgebra. In reference [8] it was shown that this result generalises to hyperbolic Kac-Moody algebras in the sense that they contain a real principal so $(1,2)$ subalgebra. However, as we shall see, this property is not just restricted to hyperbolic algebras, but holds for a wider class of Lorentzian algebras, including many of the algebras constructed in this paper.

Let us recall the discussion of reference [8], extended to include non-simply-laced Lie algebras. We define a generator $J_{3}$ by $J_{3}=-\rho^{i} H_{i}$, where $H_{i}$ are the Cartan generators in the Cartan-Weyl basis, and $\rho$ is the Weyl vector which, by definition, satisfies $\left(\rho, \alpha_{i}\right)=1$ for all simple roots $\alpha_{i}$. If we were dealing with a finite dimensional semi-simple Lie algebra, the definition of $J^{3}$ would not include a minus sign and we would find an so(3) subalgebra.

Next we recall that the fundamental weights $\lambda_{j}$ of a Kac-Moody algebra are characterised by the property

$$
\frac{2\left(\lambda_{j}, \alpha_{i}\right)}{\left(\alpha_{i}, \alpha_{i}\right)}=\delta_{i j}
$$

Given this definition, we can therefore express the Weyl vector as

$$
\rho=\sum_{i=1}^{r} \frac{2}{\left(\alpha_{i}, \alpha_{i}\right)} \lambda_{i}
$$

Furthermore, using the definition of the Cartan matrix $A_{i j}=2 \frac{\left(\alpha_{i}, \alpha_{j}\right)}{\left(\alpha_{i}, \alpha_{i}\right)}$ we find that

$$
\lambda_{i}=\sum_{j=1}^{r}\left(\alpha_{i}, \alpha_{i}\right) A_{i j}^{-1} \frac{\alpha_{j}}{\left(\alpha_{j}, \alpha_{j}\right)},
$$

and, as a result, we can express the Weyl vector as

$$
\rho=\sum_{i, j} A_{i j}^{-1} \frac{2 \alpha_{j}}{\left(\alpha_{j}, \alpha_{j}\right)} .
$$

The generator $J_{3}$ then becomes

$$
J_{3}=-\sum_{i, j} A_{i j}^{-1} H_{j}
$$

where now the $H_{j}$ are the Cartan generators in the Chevalley basis.

The remaining generators of the so $(1,2)$ algebra, denoted $J^{ \pm}$, are taken to be given by sums of the simple positive and negative root generators respectively,

$$
J^{+}=\sum_{i=1}^{r} p_{i} E_{i}, \quad J^{-}=\sum_{i=1}^{r} q_{i} F_{i},
$$


where $p_{i}, q_{i}$ will be determined shortly. Given the hermiticity property $\left(E_{i}\right)^{\dagger}=F_{i}$, the generators $J^{+}$and $J^{-}$inherit the standard hermiticity property $\left(J^{+}\right)^{\dagger}=J^{-}$provided that $p_{i}=q_{i}^{*}$. On the other hand, demanding that $\left[J^{+}, J^{-}\right]=-J^{3}$, and using the Serre relations, one finds that

$$
p_{j} q_{j}=\left|q_{j}\right|^{2}=-\sum_{i=1}^{r} A_{i j}^{-1} .
$$

The remaining relations of $\operatorname{so}(1,2)\left[J^{3}, J^{ \pm}\right]= \pm J^{ \pm}$are then automatically satisfied. Hence we conclude that a real principal so $(1,2)$ subalgebra exists if and only if

$$
\sum_{i=1}^{r} A_{i j}^{-1} \leq 0 \quad \text { for each } j
$$

We also note that it follows from equation (A.3) that

$$
A_{i j}^{-1}=\frac{2}{\left(\alpha_{i}, \alpha_{i}\right)}\left(\lambda_{i}, \lambda_{j}\right)
$$

In the following we shall only consider principal so $(1,2)$ subalgebras that satisfy the above reality property; we shall therefore drop the qualifier 'real'.

\section{Appendix B. Some properties of $\mathbf{I I}^{D-1,1}$}

Even self-dual Lorentzian lattices only exist in dimensions $D=8 n+2, n=0,1,2, \ldots$, and for each $n$, there is only one such lattice, denoted by $\mathrm{II}^{D-1,1}$. These lattices can be defined as follows. Let $x=\left(x^{1}, \ldots, x^{D-1} ; x^{0}\right) \in \mathbb{R}^{D-1,1}$, and let us denote by $r \in \mathbb{R}^{D-1,1}$ the vector $r=\left(\frac{1}{2}, \ldots, \frac{1}{2} ; \frac{1}{2}\right)$. Then $x \in \mathrm{II}^{D-1,1}$ provided that

$$
x . r \in \mathbb{Z}
$$

and, in addition, either

$$
\text { all } x^{\mu} \in \mathbb{Z} \quad \text { or } \quad \text { all } x^{\mu}-r^{\mu} \in \mathbb{Z} \text {. }
$$

In the above, the scalar product is the usual scalar product of Minkowski space, i.e.

$$
x . y=\sum_{i=1}^{D-1} x^{i} y^{i}-x^{0} y^{0}
$$

Let us now consider in detail the lattice $\mathrm{II}^{1,1}$. Using equations (B.1) and (B.2) it is straightforward to verify that $\mathrm{II}^{1,1}$ consists of the vectors

$$
(m, 2 p+m) \text { and }\left(n+\frac{1}{2}, 2 q+n+\frac{1}{2}\right) \quad \forall m, n, p, q \in \mathbb{Z} .
$$


In this paper we will use a description of the lattice $\mathrm{II}^{1,1}$ in terms of vectors $z=\left(z^{+}, z^{-}\right)$ that are related to the vectors $x$ given above by the change of basis

$$
z^{+}=x^{0}+x^{1}, \quad z^{-}=\frac{1}{2}\left(x^{0}-x^{1}\right)
$$

In terms of these vectors the scalar product becomes $x . y=-z^{+} w^{-}-z^{-} w^{+}$, where $w^{ \pm}$ are defined in terms of $y$ as in (B.5). In the basis described by $\left(z^{+}, z^{-}\right)$, the vectors of the lattice $\mathrm{II}^{1,1}$ have the simple form

$$
(n, m) \quad \forall n, m \in \mathbb{Z}
$$

The vector $r$ is now simply $r=(1,0)$.

The null vectors of $\mathrm{II}^{1,1}$ are clearly of the form $(n, 0)$ and $(0, m)$ and so the primitive null vectors can be taken to be given by $k \equiv(1,0)$ and $\bar{k} \equiv(0,-1)$. We have chosen these vectors such that $k \cdot \bar{k}=1$. Clearly, all vectors of the lattice $\mathrm{II}^{1,1}$ are of the form $p k+q \bar{k}$ where $p, q \in \mathbb{Z}$. There are only two points of length squared two in $\mathrm{II}^{1,1}$, namely $\pm(k+\bar{k})$.

\section{Appendix C. Roots and weights of the classical Lie algebras}

In this appendix we list the roots, weights, inverse Cartan matrices and some other properties of the classical finite dimensional simple Lie algebras. Recall that a finite dimensional simple Lie algebra $\mathfrak{g}$ possesses a highest root $\theta$ which can be written in terms of the simple roots $\alpha_{i}$ as

$$
\theta=\sum_{i=1}^{r} n_{i} \alpha_{i}
$$

We refer to the $n_{i}$ as the Kac labels. The Coxeter number $h(\mathfrak{g})$ is related to the height of the highest root by

$$
h(\mathfrak{g})=1+\sum_{i=1}^{r} n_{i} .
$$

The Kac labels can also be expressed as

$$
n_{i}=\frac{2\left(\theta, \lambda_{i}\right)}{\left(\alpha_{i}, \alpha_{i}\right)} .
$$

In our conventions the Cartan matrix is given by $A_{i j}=2 \frac{\left(\alpha_{i}, \alpha_{j}\right)}{\left(\alpha_{i}, \alpha_{i}\right)}$, and the fundamental weights are defined by

$$
\frac{2\left(\lambda_{j}, \alpha_{i}\right)}{\left(\alpha_{i}, \alpha_{i}\right)}=\delta_{i j}
$$


The inverse Cartan matrix can be expressed in terms of these by

$$
A_{i j}^{-1}=\frac{2}{\left(\alpha_{i}, \alpha_{i}\right)}\left(\lambda_{i}, \lambda_{j}\right)
$$

\section{C.1. The algebras $a_{r}$ or $s u(r+1)$}

Let $e_{i}, i=1, \ldots, r+1$, be a set of pairwise orthogonal unit vectors in $\mathbb{R}^{r+1}$. We can write the roots of $s u(r+1)$ as

$$
\alpha_{i}=e_{i}-e_{i+1}, \quad i=1, \ldots, r
$$

The highest root is $\theta=e_{1}-e_{r+1}=\sum_{i=1}^{r} \alpha_{i}$. Hence, the Kac labels are given by $n_{i}=1$ and the Coxeter number is $h=r+1$. The fundamental weights are given by

$$
\lambda_{i}=\sum_{j=1}^{i} e_{j}-\frac{i}{r+1} \sum_{j=1}^{r+1} e_{j}
$$

In particular, we therefore have that the Weyl vector is given by

$$
\rho=\sum_{i=1}^{r+1}\left(\frac{r+2-2 i}{2}\right) e_{i}
$$

Using equation (C.5) we find that the inverse Cartan matrix is given by

$$
A_{i j}^{-1}=i \frac{(r+1-j)}{r+1}, \quad \text { where } j \geq i
$$

Summing on the first index we find that

$$
\sum_{i=1}^{r} A_{i j}^{-1}=\frac{j}{2}(r+1-j)
$$

\section{C.2. The algebras $d_{r}$ or $s o(2 r)$}

Let $e_{i}, i=1, \ldots, r$, be a set of orthonormal vectors in $\mathbb{R}^{r}$. The roots of $s o(2 r)$ are given by

$$
\begin{aligned}
& \alpha_{i}=e_{i}-e_{i+1}, \quad i=1, \ldots, r-1, \\
& \alpha_{r}=e_{r-1}+e_{r} .
\end{aligned}
$$

The highest root is

$$
\theta=e_{1}+e_{2}=\alpha_{1}+2 \sum_{i=2}^{r-2} \alpha_{i}+\alpha_{r-1}+\alpha_{r}
$$


Hence, the Kac labels are given by $n_{i}=2, i=2, \ldots, r-2, n_{1}=n_{r-1}=n_{r}=1$, and the Coxeter number is $h=2(r-1)$. The fundamental weights are given by

$$
\begin{aligned}
\lambda_{i} & =\sum_{j=1}^{i} e_{j}, \quad i=1, \ldots, r-2, \\
\lambda_{r-1} & =\frac{1}{2} \sum_{j=1}^{r-1} e_{j}-\frac{1}{2} e_{r}, \\
\lambda_{r} & =\frac{1}{2} \sum_{j=1}^{r-1} e_{j}+\frac{1}{2} e_{r} .
\end{aligned}
$$

The Weyl vector is therefore of the form

$$
\rho=\sum_{i=1}^{r-1}(r-i) e_{i} .
$$

Using equation (C.5) we find that the inverse Cartan matrix is then

$$
\begin{aligned}
A_{i j}^{-1} & =i, & & \text { for } i \leq j, i, j=1, \ldots, r-2, \\
A_{i r-1}^{-1} & =A_{i r}^{-1}=\frac{i}{2}, & & \text { for } i=1, \ldots, r-2, \\
A_{r-1 r-1}^{-1} & =A_{r r}^{-1}=\frac{r}{4}, & & \\
A_{r-1 r}^{-1} & =\frac{r-2}{4} . & &
\end{aligned}
$$

Summing on the first index we find that

$$
\sum_{i=1}^{r} A_{i j}^{-1}=\frac{j}{2}(2 r-1-j), \quad j=1, \ldots, r-2,
$$

and

$$
\sum_{i=1}^{r} A_{i r-1}^{-1}=\sum_{i=1}^{r} A_{i r}^{-1}=\frac{r(r-1)}{4} .
$$

\section{C.3. The algebras $b_{r}$ or so $(2 r+1)$}

Let $e_{i}, i=1, \ldots, r$, be a set of orthonormal vectors in $\mathbb{R}^{r}$. The roots of $s o(2 r+1)$ are given by

$$
\begin{aligned}
\alpha_{i} & =e_{i}-e_{i+1}, \quad i=1, \ldots, r-1, \\
\alpha_{r} & =e_{r} .
\end{aligned}
$$

The highest root is

$$
\theta=e_{1}+e_{2}=\alpha_{1}+2 \sum_{i=2}^{r} \alpha_{i}
$$


Hence, the Kac labels are given by $n_{i}=2, i=2, \ldots, r$ and $n_{1}=1$, and the Coxeter number is $h=2 r$. The fundamental weights are

$$
\begin{aligned}
\lambda_{i} & =\sum_{j=1}^{i} e_{j}, \quad i=1, \ldots, r-1, \\
\lambda_{r} & =\frac{1}{2} \sum_{j=1}^{r} e_{j} .
\end{aligned}
$$

Using equation (C.5) we find that the inverse Cartan matrix is given by

$$
\begin{aligned}
A_{i j}^{-1} & =i, \quad \text { for } i \leq j, i, j=1, \ldots, r-1, \\
A_{r i}^{-1} & =i, \\
A_{i r}^{-1} & =\frac{i}{2} \\
A_{r r}^{-1} & =\frac{r}{2} .
\end{aligned}
$$

Summing on the first index we find that

$$
\begin{aligned}
& \sum_{i=1}^{r} A_{i j}^{-1}=\frac{j}{2}(2 r+1-j), \quad j=1, \ldots, r-1, \\
& \sum_{i=1}^{r} A_{i r}^{-1}=\frac{r}{4}(r+1) .
\end{aligned}
$$

C.4. The algebras $c_{r}$ or $s p(2 r)$

Let $e_{i}, i=1, \ldots, r$ be a set of orthonormal vectors in $\mathbb{R}^{r}$. The roots of $s p(2 r)$ are

$$
\begin{aligned}
\alpha_{i} & =\frac{1}{\sqrt{2}}\left(e_{i}-e_{i+1}\right), \quad i=1, \ldots, r-1, \\
\alpha_{r} & =\sqrt{2} e_{r}
\end{aligned}
$$

The highest root is

$$
\theta=\sqrt{2} e_{1}=2 \sum_{i=1}^{r-1} \alpha_{i}+\alpha_{r} .
$$

Hence, the Kac labels are given by $n_{i}=2, i=1, \ldots, r-1$ and $n_{r}=1$, and the Coxeter number is $h=2 r$. The fundamental weights are

$$
\lambda_{i}=\frac{1}{\sqrt{2}} \sum_{j=1}^{i} e_{j}, \quad i=1, \ldots, r .
$$


Using equation (C.5) we find that the inverse Cartan matrix is then

$$
\begin{aligned}
& A_{i j}^{-1}=i, \quad \text { for } j \geq i, i, j=1, \ldots, r-1, \\
& A_{i r}^{-1}=i, \\
& A_{r i}^{-1}=\frac{i}{2} \\
& A_{r r}^{-1}=\frac{r}{2} .
\end{aligned}
$$

Summing on the first index we find that

$$
\begin{aligned}
& \sum_{i=1}^{r} A_{i j}^{-1}=\frac{j}{2}(2 r-j), \quad j=1, \ldots, r-1, \\
& \sum_{i=1}^{r} A_{i r}^{-1}=\frac{r^{2}}{2} .
\end{aligned}
$$

\section{Appendix D. An explicit bound}

In section 4 we showed abstractly that only finitely many of the algebras for which there is a single link between the central node and the affine algebra $C_{1}$ admit a principal so(1,2) subalgebra. Here we want to give a more explicit bound for a certain subclass of such algebras. The subclass of algebras consists of those algebras for which the central node is linked by precisely one edge to each of the simple finite Lie algebras in $C_{2}, \ldots, C_{n}$, as well as to the affine node of $C_{1}$. All algebras are assumed to be simply-laced in this appendix.

For each $C_{p}$, let us denote the node that attaches to the central node by $s_{p}$. Then $\nu=\sum_{p=2}^{n} \lambda_{s_{p}}^{(p)}$. Let us introduce the notation

$$
X_{j}^{(p)}=\left(h\left(\mathfrak{g}_{1}\right) \nu-\rho^{(p)}\right) \cdot \lambda_{j}^{(p)}=h\left(\mathfrak{g}_{1}\right) A_{s_{p} j}^{(p)-1}-\sum_{i=1}^{r_{p}} A_{i j}^{(p)-1} .
$$

Next we consider the inequality (A.8) for the case when $j$ corresponds to one of the finite nodes of $C_{1}$. In terms of the inverse Cartan matrix of $\mathfrak{g}_{1}$, this inequality can be written as

$$
\sum_{p=2}^{n} X_{s_{p}}^{(p)} \leq 2 h\left(\mathfrak{g}_{1}\right)+1-\frac{1}{n_{j}} \sum_{i=1}^{r_{1}} A_{i j}^{(1)-1}
$$

where $j \in\left\{1, \ldots, r_{1}\right\}$ is arbitrary. Similarly, the inequality (A.8) for the case when $j$ corresponds to one of the nodes of $C_{2}, \ldots, C_{n}$ is

$$
X_{j}^{(p)} \geq 0 \quad \text { for every } j=1, \ldots, r_{p}, p=2, \ldots, n \text {. }
$$


Combining these two equations we therefore find that

$$
0 \leq 2 h\left(\mathfrak{g}_{1}\right)+1-\frac{1}{n_{j}} \sum_{i=1}^{r_{1}} A_{i j}^{(1)-1},
$$

which only depends on $\mathfrak{g}_{1}$. This inequality implies that $r_{1} \leq 15$ for $\mathfrak{g}_{1}=a_{r_{1}}$ and $r_{1} \leq 16$ for $\mathfrak{g}_{1}=d_{r_{1}}$.

Next we observe that for $\mathfrak{g}_{p}=a_{r_{p}}, X_{s_{p}}^{(p)} \geq 0$ implies that $r_{p}+1 \leq 2 h\left(\mathfrak{g}_{1}\right)$. However, the bound of equation (D.3) is valid for all $j$ and we can find stronger bounds by considering other values of $j$. In particular, if $s_{p} \leq \frac{r_{p}}{2}$, we can choose $j=2 s_{p}$, while if $s_{p} \geq \frac{r_{p}}{2}+1$, we can choose $r_{p}+1-j=2\left(r_{p}+1-s_{p}\right)$ and in both cases one finds that equation (D.3) implies the stronger result $r_{p}+1 \leq h\left(\mathfrak{g}_{1}\right)$. This in turn implies that $X_{s_{p}}^{(p)} \geq \frac{1}{2} s_{p}\left(r_{p}+1-s_{p}\right) \geq \frac{r_{p}}{2}$. This bound applies to all possible values of $s_{p}$ except the case of $s_{p}=\frac{\left(r_{p}+1\right)}{2}$ for $r_{p}$ odd for which one gets the slightly weaker bound $r_{p}-1 \leq h\left(\mathfrak{g}_{1}\right)$. Carrying out a similar analysis for $\mathfrak{g}_{p}=d_{r_{p}}$ one finds, with the exception of one value of $s_{p}$, that one can prove the same bound on $X_{s_{p}}^{(p)}$. Hence, apart from these exceptional values of $s_{p}$, the bound of equation (D.2) can be written as

$$
2+\sum_{p=1}^{n} r_{p} \leq \begin{cases}3-\frac{\left(r_{1}+1\right)\left(r_{1}-19\right)}{4}, & \text { for } \mathfrak{g}_{0}=a_{r_{1}} \\ 5-\frac{\left(r_{1}-1\right)\left(r_{1}-18\right)}{2}, & \text { for } \mathfrak{g}_{0}=d_{r_{1}}\end{cases}
$$

In deriving this equation we have made a suitable subtraction from both sides so that the the left hand side is just the total rank of the Kac-Moody algebra $C$. It then follows that the rank of the Kac-Moody algebra is bounded by 28 if $\mathfrak{g}_{0}=a_{r_{0}}$, and by 41 if $\mathfrak{g}_{0}=d_{r_{0}}$. Actually, with a little bit of extra work one can show that these bounds also apply to the exceptional values of $s_{p}$ excluded above. 


\section{References}

[1] P. Goddard, D.I. Olive, Algebras, lattices and strings, in: Vertex operators in Mathematics and Physics, MSRI Publication $\sharp 3$, Springer (1984) 51.

[2] E. Witten, String theory dynamics in various dimensions, Nucl. Phys. B443 (1995) 85 ; hep-th/9503124.

[3] P. West, Hidden superconformal symmetry in M-theory, J. High Energy Phys. 0008 (2000) 007; hep-th/0005270.

[4] P. West, $E_{11}$ and $M$-theory, Class. Quant. Grav. 18 (2001) 4443; hep-th/0104081.

[5] I. Schnakenburg, P. West, Kac-Moody symmetries of IIB supergravity, Phys. Lett. B517 (2001) 421; hep-th/0107181.

[6] W. Nahm, Supersymmetries and their representations, Nucl. Phys B135 (1978) 149.

[7] V.G. Kac, Infinite dimensional Lie algebras, Cambridge University Press (1990) [3rd ed.].

[8] H. Nicolai, D.I. Olive, The principal SO(1,2) subalgebra of a hyperbolic Kac-Moody algebra, Lett. Math. Phys. 58 (2001) 141; hep-th/0107146.

[9] V. Ruuska, On purely hyperbolic Kac-Moody algebras, in: Topological and Geometrical Methods in Field Theory, eds. J. Mickelsson and O. Pekonen, World Scientific (1992) 359.

[10] J.W.B. Hughes, Principal three dimensional subalgebras of Kac-Moody algebras, in: Infinite dimensional Lie algebras and their applications, ed. S. Kass, World Scientific (1988) 84.

[11] A.C. Aitken, Determinants and Matrices, Oliver and Boyd (1954).

[12] J.H. Conway, N.J.A. Sloane, Sphere packings, lattices and groups, Springer (1999) [3rd ed.]. 